\title{
Total Synthesis of Amaminol A: Establishment of the
}

\section{Absolute Stereochemistry}

\author{
Esa T. T. Kumpulainen ${ }^{a}$, Ari M. P. Koskinen ${ }^{*}$ and Kari Rissanen $^{\text {b\# }}$ \\ ${ }^{a}$ Laboratory of Organic Chemistry, Helsinki University of Technology, P.O.Box. 6100, FI-02015 TKK, \\ Finland \\ ${ }^{\mathrm{b}}$ NanoScience Center, Department of Chemistry, University of Jyväskylä, P.O.Box. 35, FI-40014 \\ University of Jyväskylä, Finland \\ ari.koskinen@tkk.fi
}

\section{Supporting Information}

General Methods. All reactions were carried out under an argon atmosphere in flame-dried glassware, unless otherwise noted. Nonaqueous reagents were transferred under argon via syringe or cannula and dried prior to use. THF and ether were distilled from $\mathrm{Na} /$ benzophenone, toluene from $\mathrm{Na}$, $\mathrm{MeOH}$ from $\mathrm{Mg}(\mathrm{OMe})_{2}$ and $\mathrm{CH}_{2} \mathrm{Cl}_{2}$ from $\mathrm{CaH}_{2}$. The concentration of butyl lithium was determined by double titration method using 1,3-diphenyl acetone-p-tosyl hydrazone (200 mg) in dry THF (4 ml). Other solvents and reagents were used as obtained from supplier, unless otherwise noted. Analytical TLC was performed using Merck silica gel $\mathrm{F}_{254}(10-12 \mu \mathrm{m})$ plates and analyzed by UV light or by staining upon heating either with ninhydrin solution $(1 \mathrm{~g}$ ninhydrin, $100 \mathrm{~mL}$ ethanol, 5 drops glacial acetic acid), PMA solution (2.5 g phosphomolybdic acid, $100 \mathrm{~mL}$ EtOH, $5 \mathrm{~mL}$ conc. $\mathrm{H}_{2} \mathrm{SO}_{4}, 1.5 \mathrm{~mL}$

\footnotetext{
${ }^{\#}$ X-ray crystallography.
} 
$85 \% \mathrm{H}_{3} \mathrm{PO}_{4}$ ) or $\mathrm{KMnO}_{4}$ solution (1 $\mathrm{g}^{\mathrm{KMnO}_{4}, 2} \mathrm{~g} \mathrm{Na}_{2} \mathrm{CO}_{3}, 100 \mathrm{~mL} \mathrm{H}_{2} \mathrm{O}$ ). For silica gel chromatography, the flash chromatography technique was used, with Merck silica gel $60(40-63 \mu \mathrm{m})$ and p.a. grade solvents unless otherwise noted.

IR spectra were recorded on a Perkin-Elmer Spectrum One spectrometer. Optical rotations were obtained with a Perkin-Elmer 343 polarimeter. The ${ }^{1} \mathrm{H}$ NMR and ${ }^{13} \mathrm{C}$ NMR spectra were recorded in $\mathrm{CDCl}_{3}$ or in $d 4-\mathrm{MeOD}$ on a Bruker Avance $400\left({ }^{1} \mathrm{H} 399.98 \mathrm{MHz} ;{ }^{13} \mathrm{C} 100.59 \mathrm{MHz}\right)$ spectrometer. The chemical shifts are reported in ppm relative to residual $\mathrm{CHCl}_{3}(\delta 7.26)$ or residual $\mathrm{MeOH}(\delta 3.31)$ for ${ }^{1} \mathrm{H}$ NMR. For the ${ }^{13} \mathrm{C}$ NMR spectra, the solvent peaks $\mathrm{CDCl}_{3}(\delta 77.0)$ and $d 4-\mathrm{MeOD}(\delta 49.0)$ were used as the internal standard. HRMS-spectra were recorded on Waters LCT Premier -spectrometer. The enantiomeric excess (ee) of the IMDA products were determined by GC in comparison to the corresponding racemic samples using a Hewlett Packard HP 5890 instrument, Supelco $\beta$-Dex ${ }^{\mathrm{TM}} 120$ column $\left(30 \mathrm{~m} \times 0.25 \mathrm{~mm}, 0.25 \mu \mathrm{m}\right.$ film) in isotherm $\left(130{ }^{\circ} \mathrm{C}\right)$, helium as carrier gas $(34 \mathrm{~cm} / \mathrm{s})$, with Hewlett Packard $5971 \mathrm{MS}$ detector $\left(270{ }^{\circ} \mathrm{C}\right)$ for ee analysis and isotherm $\left(145^{\circ} \mathrm{C}\right)$, helium as carrier gas $(33 \mathrm{~cm} / \mathrm{s})$ for de analysis.

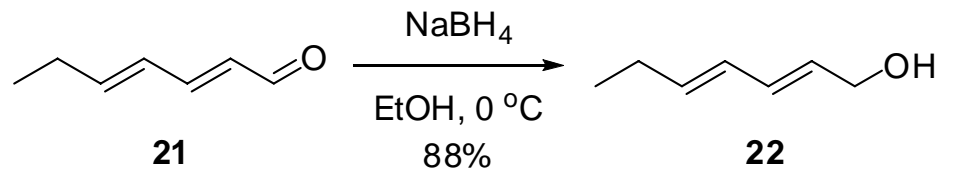

(2E,4E)-heptadien-1-ol (22). ${ }^{1}$ Heptadienal 21 (14.2 mL, $100 \mathrm{mmol}, 100 \mathrm{~mol} \%$, Kosher grade >88\%) was dissolved in EtOH $(200 \mathrm{~mL})$ and cooled to $0{ }^{\circ} \mathrm{C}$. Sodium borohydride $(3.9 \mathrm{~g}, 100 \mathrm{mmol}, 100 \mathrm{~mol}$ $\%$ ) was added and the mixture was stirred for $15 \mathrm{~min}$. The solution was concentrated and the residue partitioned between ether $(200 \mathrm{~mL})$ and aqueous $1 \mathrm{M} \mathrm{NaOH}(200 \mathrm{~mL})$. The aqueous phase was backextracted with ether $(100 \mathrm{~mL})$. The combined organic extracts were washed with brine $(100 \mathrm{~mL})$, dried over anhydrous sodium sulfate and evaporated to give a yellow oil. The product was purified by distillation under reduced pressure $(18 \mathrm{mmHg})$ to give alcohol 13 (9.90 g, 88\%) as a clear liquid. B.p. 
96-98 ${ }^{\circ} \mathrm{C}(18 \mathrm{mmHg}) . R_{f}=0.19(20 \%$ EtOAc $/$ hexanes $) ;{ }^{1} \mathrm{H}-\mathrm{NMR}\left(400 \mathrm{MHz}, \mathrm{CDCl}_{3}\right) \delta 6.22(\mathrm{dd}, J=$ 10.1, 15.1 Hz, 1H), $6.04(\mathrm{dd}, J=10.2,15.0 \mathrm{~Hz}, 1 \mathrm{H}), 5.68-5.80(\mathrm{~m}, 2 \mathrm{H}), 4.16(\mathrm{t}, J=5.6 \mathrm{~Hz}, 2 \mathrm{H}), 2.10$ $(\mathrm{dq}, J=6.4,7.1 \mathrm{~Hz}, 2 \mathrm{H}), 1.34(\mathrm{t}, J=5.8 \mathrm{~Hz}, 1 \mathrm{H}), 1.01(\mathrm{t}, J=7.4 \mathrm{~Hz}, 3 \mathrm{H}) ;{ }^{13} \mathrm{C}-\mathrm{NMR}\left(100 \mathrm{MHz}, \mathrm{CDCl}_{3}\right)$ $\delta 137.2,132.1,129.3,128.3,63.5,25.6,13.4$.

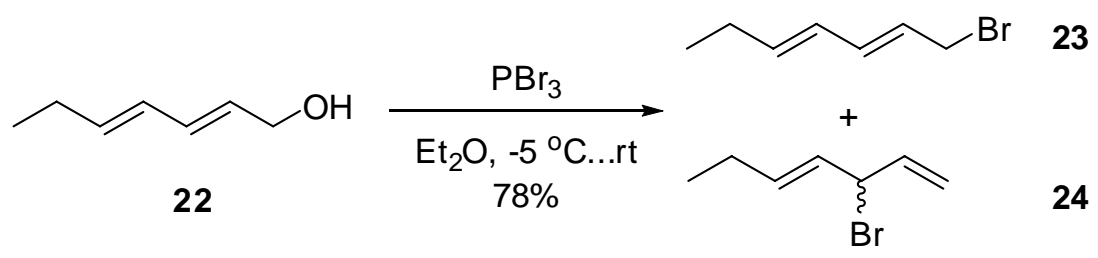

(2E,4E)-1-Bromohepta-2,4-diene (23) and $(E)$-3-bromohepta-1,4-diene (24). A slurry of $(2 E, 4 E)$ hepta-2,4-dienol 22 (3.20 mL, $25 \mathrm{mmol}, 100 \mathrm{~mol} \%)$ and calcium hydride (1.57 g, $37.5 \mathrm{mmol}, 150 \mathrm{~mol}$ $\%)$ in dry ether $(20 \mathrm{~mL})$ was stirred for 60 minutes. The reaction mixture was cooled to $0^{\circ} \mathrm{C}$ and phosphorous tribromide $(0.87 \mathrm{~mL}, 9.3 \mathrm{mmol}, 37.3 \mathrm{~mol} \%)$ in dry ether $(5 \mathrm{~mL})$ was added. After 40 minutes the reaction was quenched by addition of methanol $(0.15 \mathrm{~mL}, 3.7 \mathrm{mmol}, 15 \mathrm{~mol} \%)$ after which the mixture was allowed to warm to room temperature. The mixture was filtered through celite followed by ether washings. The solvent was evaporated and the crude product purified by Kugelrohr distillation $\left(15 \mathrm{mmHg}\right.$, air bath $\left.80{ }^{\circ} \mathrm{C}\right)$ to afford a $2: 1$ mixture of bromides 23 and $\mathbf{2 4}(3.44 \mathrm{~g}, 78 \%)$ as a slightly yellow oil. The product decomposes at room temperature but it was found to be only slightly decomposed when kept four months in a freezer $\left(-18^{\circ} \mathrm{C}\right) . R_{f}=0.67$ (20\% EtOAc / hexanes).

For 23: ${ }^{1} \mathrm{H}-\mathrm{NMR}\left(400 \mathrm{MHz}, \mathrm{CDCl}_{3}\right) \delta$ 6.16-6.37 (m, 1H), $6.03(\mathrm{dd}, J=10.4,15.2 \mathrm{~Hz}, 1 \mathrm{H}), 5.71-5.89$ $(\mathrm{m}, 2 \mathrm{H}), 4.03(\mathrm{~d}, J=7.8 \mathrm{~Hz}, 2 \mathrm{H}), 2.12(\mathrm{dq}, J=6.3,7.0 \mathrm{~Hz}, 2 \mathrm{H}), 1.01(\mathrm{t}, J=7.4 \mathrm{~Hz}, 3 \mathrm{H}) ;{ }^{13} \mathrm{C}-\mathrm{NMR}$ $\left(100 \mathrm{MHz}, \mathrm{CDCl}_{3}\right) \delta 139.1,135.3,127.8,126.1,33.8,25.6,13.2$.

For 24: ${ }^{1} \mathrm{H}-\mathrm{NMR}\left(400 \mathrm{MHz}, \mathrm{CDCl}_{3}\right) \delta 5.26(\mathrm{dd}, J=1.2 \mathrm{~Hz}, 16.5 \mathrm{~Hz}, 1 \mathrm{H}), 5.15(\mathrm{dd}, J=1.2,9.7 \mathrm{~Hz}$, $1 \mathrm{H}), 4.51(\mathrm{dt}, J=6.8,9.4 \mathrm{~Hz}, 1 \mathrm{H}), 1.86-2.05(\mathrm{~m}, 2 \mathrm{H}), 1.00(\mathrm{t}, J=7.3 \mathrm{~Hz})$. Other proton signals were obscured; ${ }^{13} \mathrm{C}-\mathrm{NMR}\left(100 \mathrm{MHz}, \mathrm{CDCl}_{3}\right) \delta 135.6,134.3,132.4,119.0,57.5,32.3,12.3$. 


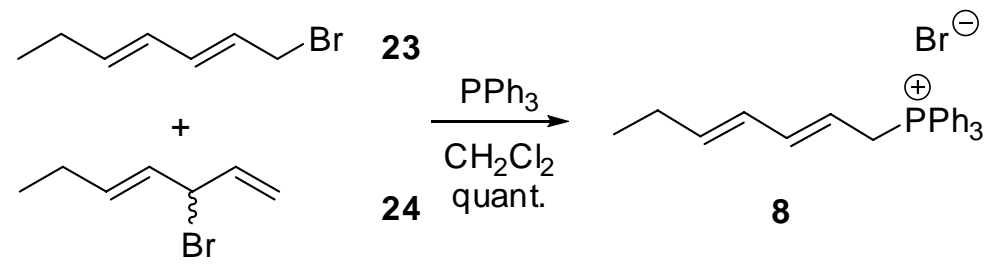

(2E,4E)-Hepta-2,4-dienyltriphenylphosphonium bromide (8). ${ }^{2}$ Triphenyl phosphine (8.66 g, 33.0 mmol, $100 \mathrm{~mol} \%)$ was dissolved in dry dichloromethane $(85 \mathrm{~mL})$ and a mixture of bromides $\mathbf{2 3}$ and $\mathbf{2 4}$ (6.07 g, $34.7 \mathrm{mmol}, 105 \mathrm{~mol} \%)$ was added at room temperature. The mixture was stirred for 20 hours and the solvent was evaporated and the residue dried in vacuo for 14 hours to give $14.78 \mathrm{~g}$ (quant.) of phosphonium salt $\mathbf{6}$ as white foam. The product is highly hygroscopic, light sensitive and decomposes at room temperature and therefore it was used as such. It was found to be stable in a freezer $\left(-18^{\circ} \mathrm{C}\right) .{ }^{1} \mathrm{H}-$ $\operatorname{NMR}\left(400 \mathrm{MHz}, \mathrm{CDCl}_{3}\right) \delta 7.64-7.89(\mathrm{~m}, 15 \mathrm{H}), 6.37(\mathrm{ddd}, J=5.1,10.2,15.1 \mathrm{~Hz}, 1 \mathrm{H}), 5.89(\mathrm{dd}, J=$ $10.8,14.7 \mathrm{~Hz}, 1 \mathrm{H}), 5.69$ (ddt, $J=2.4,4.8,11.9 \mathrm{~Hz}, 1 \mathrm{H}), 5.27-5.38(\mathrm{~m}, 1 \mathrm{H}), 4.75(\mathrm{dd}, J=7.4,15.2 \mathrm{~Hz}$, 2H), $2.03(\mathrm{dq}, J=7.5,7.7 \mathrm{~Hz}, 2 \mathrm{H}), 0.94(\mathrm{t}, J=7.4 \mathrm{~Hz}, 3 \mathrm{H}) ;{ }^{13} \mathrm{C}-\mathrm{NMR}\left(100 \mathrm{MHz}, \mathrm{CDCl}_{3}\right) \delta[140.9$, 140.7], [139.5, 139.4], [134.9, 134.9], [133.9, 133.8], [130.2, 130.1], [127.5, 127.4], [118.4, 117.6], [113.3, 113.1], [28.4, 28.9], [25.8, 25.4], [13.0, 13.0].

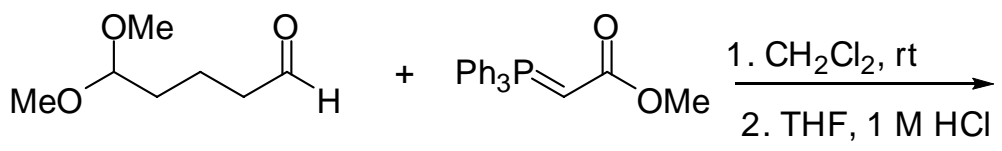

9
10

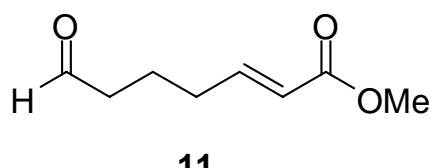

11

(E)-Methyl-7-oxo-hept-2-enoate (11). Aldehyde $\mathbf{9}^{3}$ (20.5 g, $122.0 \mathrm{mmol}, 100 \mathrm{~mol} \%$, purity $\left.~ 87 \%\right)$ was dissolved in dry dichloromethane $(280 \mathrm{~mL})$ at room temperature and methyl (triphenylphosphoranylidene)acetate $\mathbf{1 0}$ was added. The mixture was stirred overnight and the solvent was removed by evaporation. The residual white solid containing oil was treated with hexane $(100 \mathrm{~mL})$. The solids were filtered off and washed with hexane $(2 \times 50 \mathrm{~mL})$. The combined solvents were evaporated to give $29.76 \mathrm{~g}$ of crude acetal as a clear oil. 
This crude acetal was dissolved in THF $(10 \mathrm{~mL})$ and aqueous $1 \mathrm{M} \mathrm{HCl}(150 \mathrm{~mL})$. The mixture was strirred for $30 \mathrm{~min}$ and ether $(150 \mathrm{~mL})$ was added. The aqueous phase was extracted with ether $(3 \times 100$ $\mathrm{mL})$. The combined organic extracts were washed with brine $(200 \mathrm{~mL})$, dried over anhydrous sodium sulfate and evaporated to dryness. The crude product was purified by distillation under reduced pressure (0.2 mbar, $\left.70-71^{\circ} \mathrm{C}\right)$ to afford aldehyde $\mathbf{1 1}$ as a clear liquid (16.9 g, 88\% over two steps). Geometrical isomer-ratio was found to be 20:1 (E:Z). $R_{f}=0.42$ (50\% EtOAc / hexanes); ${ }^{1} \mathrm{H}-\mathrm{NMR}(400 \mathrm{MHz}$, $\left.\mathrm{CDCl}_{3}\right) \delta 9.78(\mathrm{t}, J=1.4 \mathrm{~Hz}, 1 \mathrm{H}), 6.92(\mathrm{dt}, J=6.9,15.6 \mathrm{~Hz}, 1 \mathrm{H}), 5.85(\mathrm{dt}, J=1.5,15.6 \mathrm{~Hz}, 1 \mathrm{H}), 3.73$ (s, 3H), $2.49(\mathrm{dt}, J=1.3,7.2 \mathrm{~Hz}, 2 \mathrm{H}), 2.25(\mathrm{ddt}, J=1.3,7.2 \mathrm{~Hz}, 7.3 \mathrm{~Hz}, 2 \mathrm{H}), 1.81(\mathrm{ddt}, J=7.3,7.3,7.3$ $\mathrm{Hz}) ;{ }^{13} \mathrm{C}-\mathrm{NMR}\left(100 \mathrm{MHz}, \mathrm{CDCl}_{3}\right) \delta 201.5,166.8,147.8,121.8,51.4,42.9,31.2,20.3$. These data match those reported in the literature. ${ }^{4}$

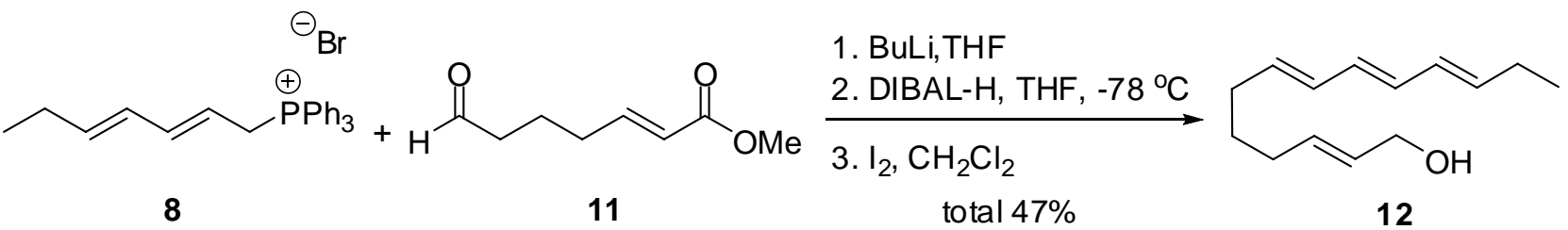

(2E,7E,9E,11E)-Tetradeca-2,7,9,11-tetraen-1-ol (12). Phosphonium salt 8 (14.4 g, 30.0 mmol, 120 mol \%, purity estimated to be $\sim 90 \%)$ in dry $\mathrm{CH}_{2} \mathrm{Cl}_{2}(20 \mathrm{~mL})$ and THF $(80 \mathrm{~mL})$ was cooled to $-30{ }^{\circ} \mathrm{C}$. To this slurry was added $2.3 \mathrm{M}$ butyl lithium in hexanes during 15 minutes $(13.0 \mathrm{~mL}, 30.0$ mmol, $120 \mathrm{~mol} \%)$ resulting in a red slurry. The mixture was stirred for $1 \mathrm{~h}$ and aldehyde $\mathbf{1 1}(3.9 \mathrm{~g}, 25.0$ mmol, $100 \mathrm{~mol} \%)$ in dry THF $(25 \mathrm{~mL})$ was cannulated into reaction mixture. The mixture was allowed to warm to room temperature and stirring was continued for $1 \mathrm{~h}$. The reaction was quenched by addition of saturated ammonium chloride solution $(80 \mathrm{~mL})$. Also $\mathrm{CH}_{2} \mathrm{Cl}_{2}(80 \mathrm{~mL})$ was added and phases were separated. The aqueous phase was extracted with $\mathrm{CH}_{2} \mathrm{Cl}_{2}(50 \mathrm{~mL})$. The combined organic extracts were washed with brine $(150 \mathrm{~mL})$, dried over anhydrous sodium sulfate and evaporated to dryness. The crude product was dissolved in a small amount of $\mathrm{CH}_{2} \mathrm{Cl}_{2}$ and filtered through a $3 \mathrm{~cm}$ pad of silica using $20 \%$ ethyl acetate in hexanes. The solvents were evaporated and residual solids containing oil was treated 
with hexanes $(100 \mathrm{~mL})$. The solids were filtered off and rinsed with hexanes $(2 \times 50 \mathrm{~mL})$. Evaporation of solvents gave $5.1 \mathrm{~g}$ of crude ester as a yellow oil. Geometrical isomer-ratio was found to be 2:1 $(E: Z)$.

The crude ester was dissolved in dry THF $(100 \mathrm{~mL})$ and cooled to $-78^{\circ} \mathrm{C}$. To this solution was added 1.0 M diisobutyl aluminium hydride in toluene $(75.0 \mathrm{~mL}, 75.0 \mathrm{mmol}, 300 \mathrm{~mol} \%)$ and the mixture was stirred for $1 \mathrm{~h}$. The reaction was quenched by addition of aqueous saturated solution of Rochelle's salt $(100 \mathrm{~mL})$ and the mixture was allowed to warm to room temperature. After $18 \mathrm{~h}$ the phases were separated and the aqueous phase was extracted with ether $(100 \mathrm{~mL})$. The combined organic extracts were dried over anhydrous sodium sulfate and evaporated to dryness. The crude product was purified by flash chromatography using $20 \%$ ethyl acetate in hexanes to give $2.51 \mathrm{~g}$ of alcohol 12 as a clear oil.

Alcohol 12 was dissolved in dichloromethane $(50 \mathrm{~mL})$ and treated with iodine $(0.15 \mathrm{~g}, 0.60 \mathrm{mmol}, 5$ mol \%). The mixture was stirred for 30 minutes and saturated solution of sodium thiosulphate $(50 \mathrm{~mL})$ was added. The biphasic mixture was stirred vigorously for 30 minutes. The phases were separated and the aqueous phase was extracted with dichloromethane $(25 \mathrm{~mL})$. The combined organic extracts were dried over anhydrous sodium sulfate and evaporated to dryness to give alcohol 12 (2.46 g, $47 \%$ over two steps) in 4:1 (E:Z) ratio as a yellow oil. $R_{f}=0.20$ (20\% EtOAc / hexanes); IR (thin film, $\left.\mathrm{cm}^{-1}\right) 3338$, 3012, 2962, 2929, 2855, 1455, 1437, 1088, 993, 968. For major isomer 12: ${ }^{1} \mathrm{H}-\mathrm{NMR}\left(400 \mathrm{MHz}, \mathrm{CDCl}_{3}\right)$ $\delta$ 5.92-6.22 (m, 4H), 5.57-5.79 (m, 4H), $4.08(\mathrm{br} \mathrm{s}, 2 \mathrm{H}), 2.01-2.26(\mathrm{~m}, 6 \mathrm{H}), 1.48$ [dq, $J=7.5 \mathrm{~Hz},(\mathrm{~d})$, 2H], $1.28($ br s, $1 \mathrm{H}), 1.00(\mathrm{t}, J=7.54 \mathrm{~Hz}) ;{ }^{13} \mathrm{C}-\mathrm{NMR}\left(100 \mathrm{MHz}, \mathrm{CDCl}_{3}\right) \delta$ 136.0, 133.6, 132.8, 131.0, $130.8,130.6,129.4,129.2,32.1,31.5,28.6,25.7,13.5$; HRMS (ESI) calc. for $[\mathrm{M}+\mathrm{Na}] \mathrm{C}_{14} \mathrm{H}_{22} \mathrm{ONa}$ 229.1568, found 229.1517, $\Delta=1.3 \mathrm{ppm}$. 


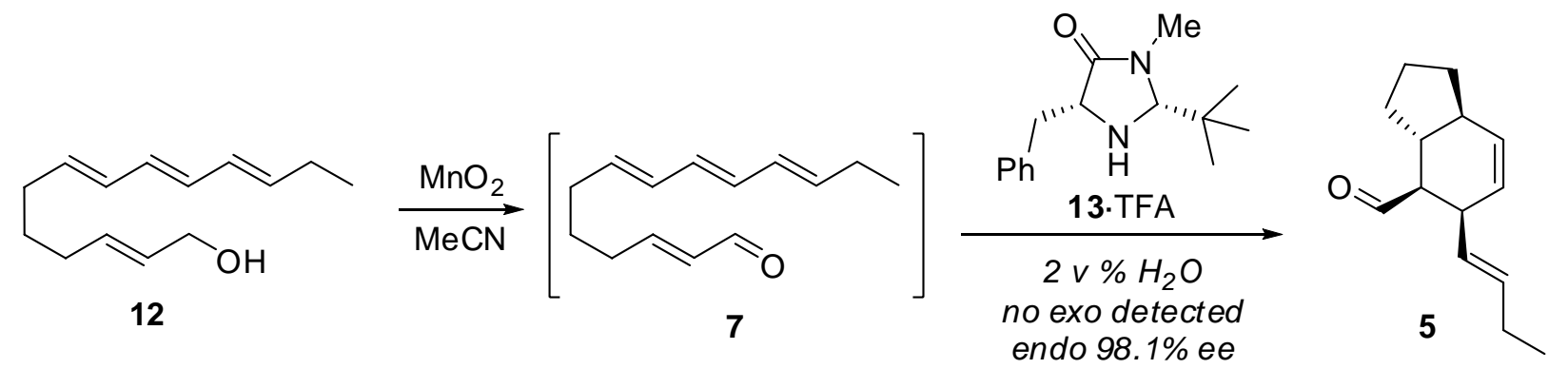

(3aR,4S,5R,7aS)-5-[(E)-But-1-enyl]-2,3,3a,4,5,7a-hexahydro-1H-indene-4-carbaldehyde

(5).

Alcohol $12(1.03 \mathrm{~g}, 5.0 \mathrm{mmol}, 100 \mathrm{~mol} \%)$ was dissolved in acetonitrile $(40 \mathrm{~mL})$ and manganese oxide (4.35 g, $50.0 \mathrm{mmol}, 1000 \mathrm{~mol} \%$ ) was added. The mixture was stirred for 1 hour at room temperature. A second portion of manganese oxide $(3.44 \mathrm{~g}, 39.5 \mathrm{mmol}, 790 \mathrm{~mol} \%)$ was added. After 5 hours, the mixture was filtered through celite followed by $\mathrm{MeCN}$ rinsing $(2 \times 10 \mathrm{~mL})$. The resulting yellow solution containing aldehyde 7 was cooled to $-20^{\circ} \mathrm{C}$ and distilled water $(1 \mathrm{~mL})$ was added. Catalyst $\mathbf{1 3}$ $(0.25 \mathrm{~g}, 1.0 \mathrm{mmol}, 20 \mathrm{~mol} \%)$ and trifluoroacetic acid $(77 \mu \mathrm{L}, 1.0 \mathrm{mmol}, 20 \mathrm{~mol} \%)$ were added and the mixture was stirred at $-18{ }^{\circ} \mathrm{C}$ for 18 hours. The reaction was quenched by addition of $1 \%$ aq. $\mathrm{NaHCO}_{3}$ $(100 \mathrm{~mL})$. Pentane $(60 \mathrm{~mL})$ was added and the mixture was allowed to warm to room temperature. The aqueous phase was extracted with pentane $(2 \times 50 \mathrm{~mL})$. The combined organic extracts were washed with brine $(100 \mathrm{~mL})$, dried over anhydrous sodium sulfate and evaporated carefully $(>300 \mathrm{mmHg}$, water bath $25^{\circ} \mathrm{C}$ ) to dryness to give $0.90 \mathrm{~g}$ of a yellow oil. The crude product was purified by flash chromatography using $5 \%$ ether in pentane to afford aldehyde $\mathbf{5}(0.36 \mathrm{~g}, 35 \%$ over two steps $)$ as a clear oil. GC-MS analysis showed >308:1 endo:exo (no exo detected) and 98.1\% ee selectivities. The product is highly volatile. $R_{f}=0.54$ (20\% EtOAc / hexanes); IR (thin film, $\left.\mathrm{cm}^{-1}\right) 3018,2959,2871,1723,1455$, 1066, 968; $[\alpha]^{20}=-223.8\left(c 1.0, \mathrm{CHCl}_{3}\right) ;{ }^{1} \mathrm{H}-\mathrm{NMR}\left(400 \mathrm{MHz}, \mathrm{CDCl}_{3}\right) \delta 9.64(\mathrm{~d}, J=2.9 \mathrm{~Hz}, 1 \mathrm{H}), 5.90$ (d, $J=9.8 \mathrm{~Hz}, 1 \mathrm{H}), 5.55(\mathrm{dt}, J=6.3,15.1 \mathrm{~Hz}, 1 \mathrm{H}), 5.43(\mathrm{ddd}, J=2.6,4.1,9.7 \mathrm{~Hz}, 1 \mathrm{H}), 5.33$ (ddt, $J=$ $1.4,8.7,15.2 \mathrm{~Hz}, 1 \mathrm{H}), 3.28-3.38(\mathrm{~m}, 1 \mathrm{H}), 2.53(\mathrm{ddd}, J=2.9,6.3,11.4 \mathrm{~Hz}, 1 \mathrm{H}), 1.95-2.04(\mathrm{~m}, 3 \mathrm{H}), 1.61-$ $1.92(\mathrm{~m}, 5 \mathrm{H}), 1.06-1.24(\mathrm{~m}, 2 \mathrm{H}), 0.94(\mathrm{t}, J=7.5 \mathrm{~Hz}, 3 \mathrm{H}) ;{ }^{13} \mathrm{C}-\mathrm{NMR}\left(100 \mathrm{MHz}, \mathrm{CDCl}_{3}\right) \delta 204.8,135.3$, 
129.7, 129.3, 127.6, 56.1, 44.9, 41.1, 39.5, 28.4, 27.5, 25.4, 22.3, 13.6; HRMS (ESI) calc. for [M+Na] $\mathrm{C}_{14} \mathrm{H}_{20} \mathrm{ONa} 227.1412$, found 227.1410, $\Delta=0.9 \mathrm{ppm}$.

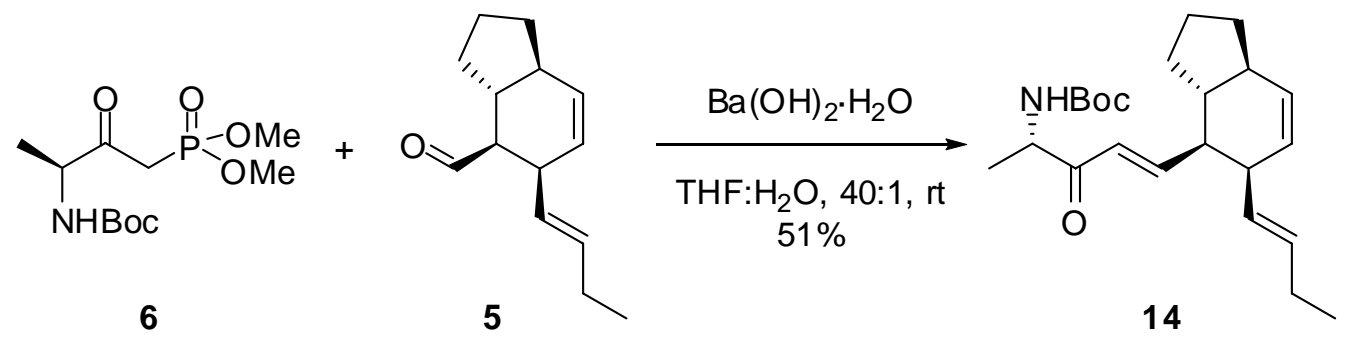

\section{tert-Butyl $\quad(S, E)-5-\{(3 a R, 4 S, 5 R, 7 a S)-5-[(E)$-but-1-enyl]-2,3,3a,4,5,7a-hexahydro-1H-inden-4-yl $\}$ -}

3-oxopent-4-en-2-ylcarbamate (14). Activated (kept 1 hour under high vacuo at 130-140 ${ }^{\circ} \mathrm{C}$ ) barium hydroxide monohydrate $(0.156 \mathrm{~g}, 0.91 \mathrm{mmol}, 55 \mathrm{~mol} \%)$ was mixed with phosphonate $6^{5}(0.537 \mathrm{~g}, 1.82$ mmol, $110 \mathrm{~mol} \%)$ in THF $(10 \mathrm{~mL})$ for 10 minutes. To this mixture was added aldehyde $\mathbf{5}(0.338,1.65$ mmol, $100 \mathrm{~mol} \%)$ in 40:1 THF: $\mathrm{H}_{2} \mathrm{O}(10 \mathrm{~mL})$ and the mixture was stirred at room temperature for 24 hours. The reaction mixture was diluted with ether $(20 \mathrm{~mL})$ and $0.5 \mathrm{M}$ phosphoric acid $(20 \mathrm{~mL})$ was added. The mixture was stirred vigorously until clear. Phases were separated and the aqueous phase was extracted with ether $(20 \mathrm{~mL})$. The combined organic extracts were washed with brine $(50 \mathrm{~mL})$, dried over anhydrous sodium sulfate and evaporated to dryness. The crude product was purified by flash chromatography using $20 \%$ ether in hexanes to give enone $14(0.317 \mathrm{~g}, 51 \%)$ as a clear oil. $R_{f}=0.47$ (20\% EtOAc / hexanes); IR (thin film, $\mathrm{cm}^{-1}$ ) 3426, 2962, 2871, 1716, 1695, 1627, 1492, 1446, 1366, 1167, 1044, 1019; $[\alpha]^{20}=-151.6\left(c\right.$ 1.0, $\left.\mathrm{CHCl}_{3}\right) ;{ }^{1} \mathrm{H}-\mathrm{NMR}\left(400 \mathrm{MHz}, \mathrm{CDCl}_{3}\right) \delta 6.87(\mathrm{dd}, J=9.9,15.8$ $\mathrm{Hz}, 1 \mathrm{H}), 6.09(\mathrm{~d}, J=15.9 \mathrm{~Hz}, 1 \mathrm{H}), 5.88(\mathrm{~d}, J=9.7 \mathrm{~Hz}, 1 \mathrm{H}), 5.21-5.53(\mathrm{~m}, 4 \mathrm{H}), 4.65(\mathrm{dq}, J=9.9,15.8$ $\mathrm{Hz}, 1 \mathrm{H}), 2.49$ [ddd, $J=6.5,10.5 \mathrm{~Hz}(\mathrm{~d}), 1 \mathrm{H}], 1.96-2.06(\mathrm{~m}, 2 \mathrm{H}), 1.79-1.92(\mathrm{~m}, 2 \mathrm{H}), 1.47-1.77(\mathrm{~m}, 3 \mathrm{H})$, $1.44(\mathrm{~s}, 9 \mathrm{H}), 1.32(\mathrm{~d}, J=7.1 \mathrm{~Hz}, 3 \mathrm{H}), 1.12-1.30(\mathrm{~m}, 1 \mathrm{H}), 0.99-1.10(\mathrm{~m}, 1 \mathrm{H}), 0.96(\mathrm{t}, J=7.4 \mathrm{~Hz}, 3 \mathrm{H})$; ${ }^{13} \mathrm{C}-\mathrm{NMR}\left(100 \mathrm{MHz}, \mathrm{CDCl}_{3}\right) \delta 198.5,155.0,152.4,134.9,129.9,129.5,128.1,126.4,79.4,52.5,48.1$, 45.2, 45.1, 42.8, 28.9, 28.3, 27.9, 25.5, 22.0, 19.5, 13.7; HRMS (ESI) calc. for [M+Na] $\mathrm{C}_{23} \mathrm{H}_{35} \mathrm{NO}_{3} \mathrm{Na}$ 396.2515, found 396.2516, $\Delta=0.3 \mathrm{ppm}$. 

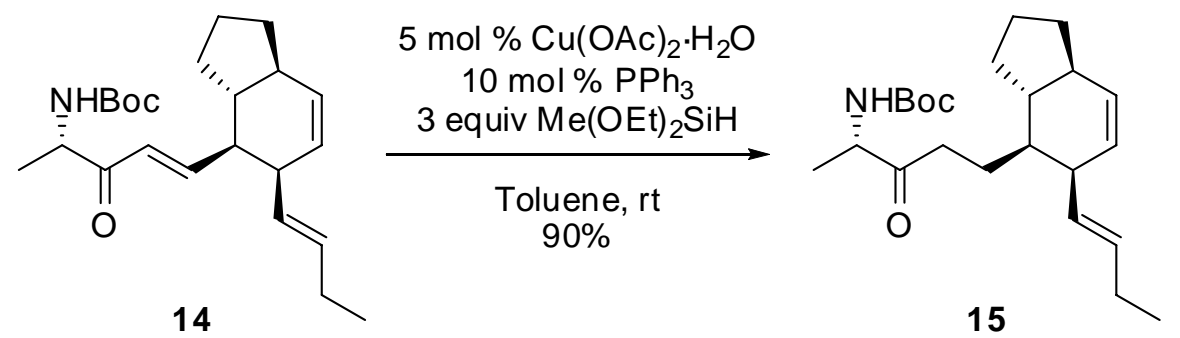

\section{tert-Butyl $(S)-5-\{(3 a R, 4 S, 5 R, 7 a S)-5-[(E)$-but-1-enyl]-2,3,3a,4,5,7a-hexahydro-1H-inden-4-yl\}-3-}

oxopentan-2-ylcarbamate (15). A flask was charged with $\mathrm{Cu}(\mathrm{OAc})_{2} \cdot \mathrm{H}_{2} \mathrm{O}(1.3 \mathrm{mg}, 6.7 \mu \mathrm{mol}, 5 \mathrm{~mol} \%$, kept $1 \mathrm{~h}$ under high vacuum at $\left.90{ }^{\circ} \mathrm{C}\right)$, dry toluene $(1 \mathrm{~mL})$ and $\mathrm{Me}(\mathrm{OEt})_{2} \mathrm{SiH}(0.056 \mathrm{~g}, 0.40 \mathrm{mmol}, 300$ mol \%) under argon. The mixture was stirred for $2 \mathrm{~h}$ while the color of the reaction mixture turned from blue to green and gradually orange. Enone $14(0.050 \mathrm{~g}, 0.13 \mathrm{mmol}, 100 \mathrm{~mol} \%)$ dissolved in dry toluene $(1 \mathrm{~mL})$ was added and mixture was stirred for $16 \mathrm{~h}$. The reaction was quenched with addition of acetic acid $(38 \mu \mathrm{L}, 0.67 \mathrm{mmol}, 500 \mathrm{~mol} \%)$ and $1 \mathrm{M}$ TBAF solution in THF $(0.20 \mathrm{~mL}, 0.20 \mathrm{mmol}, 150 \mathrm{~mol}$ $\%)$. After 20 minutes the solvents were evaporated. The crude product was purified by flash chromatography using $10-15 \%$ ether in hexanes to afford ketone $15(0.045 \mathrm{~g}, 90 \%)$ as a clear oil. $R_{f}=$ 0.42 (20\% EtOAc / hexanes); IR (thin film, $\mathrm{cm}^{-1}$ ) 3359, 2958, 2928, 2869, 1711, 1494, 1454, 1366, 1247,$1170 ;[\alpha]^{20}=-137.6\left(c\right.$ 0.9, $\left.\mathrm{CHCl}_{3}\right) ;{ }^{1} \mathrm{H}-\mathrm{NMR}\left(400 \mathrm{MHz}, \mathrm{CDCl}_{3}\right) \delta 5.80(\mathrm{~d}, J=9.6 \mathrm{~Hz}, 1 \mathrm{H}), 5.47$ $(\mathrm{dt}, J=6.3,15.1 \mathrm{~Hz}, 1 \mathrm{H}), 5.38(\mathrm{ddd}, J=2.6,4.3,9.7 \mathrm{~Hz}, 1 \mathrm{H}), 5.20-5.32(\mathrm{~m}, 2 \mathrm{H}), 4.23-4.36(\mathrm{~m}, 1 \mathrm{H})$, 2.78-2.87 (m, 1H), $2.57(\mathrm{ddd}, J=5.1,10.7,16.3 \mathrm{~Hz}, 1 \mathrm{H}), 2.39(\mathrm{ddd}, J=6.0,9.7,15.9 \mathrm{~Hz}, 1 \mathrm{H}), 2.00$ (ddq, $J=1.4,7.5,13.7 \mathrm{~Hz}, 2 \mathrm{H}), 1.61-1.86(\mathrm{~m}, 5 \mathrm{H}), 1.45-1.60(\mathrm{~m}, 2 \mathrm{H}), 1.42(\mathrm{~s}, 9 \mathrm{H}), 1.20-1.33$ (obs m, 2H), 1.30 (obs d, $J=7.1 \mathrm{~Hz}, 3 \mathrm{H}), 1.07-1.20(\mathrm{~m}, 2 \mathrm{H}), 0.95(\mathrm{t}, J=7.4 \mathrm{~Hz}, 3 \mathrm{H}) ;{ }^{13} \mathrm{C}-\mathrm{NMR}(100 \mathrm{MHz}$, $\left.\mathrm{CDCl}_{3}\right) \delta 209.8,155.1,133.8,130.7,129.4,128.3,79.5,54.7,46.1,44.6,43.2,42.0,36.5,29.2,28.3$, 27.8, 25.6, 25.0, 22.1, 18.1, 13.9; HRMS (ESI) calc. for $[\mathrm{M}+\mathrm{Na}] \mathrm{C}_{23} \mathrm{H}_{37} \mathrm{NO}_{3} \mathrm{Na}$ 398.2671, found $398.2672, \Delta=0.3 \mathrm{ppm}$. 


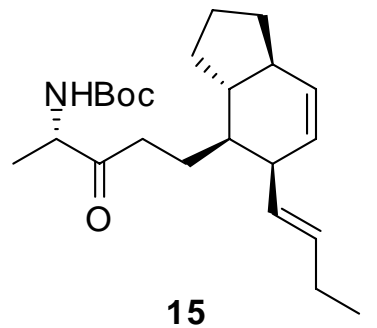

15

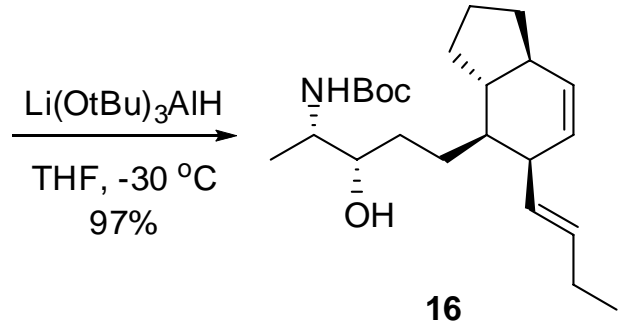

16

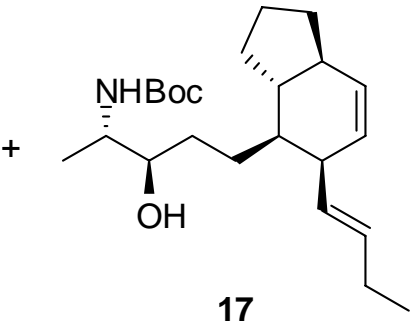

17

$N$-Boc (2S,3R)-epi-amaminol A (30) and $N$-Boc amaminol A (29). Ketone 15 (0.045 g, 0.12 mmol, $100 \mathrm{~mol} \%)$ was dissolved in dry THF $(2 \mathrm{~mL})$. The solution was cooled to $-30{ }^{\circ} \mathrm{C}$ and lithium tri-tertbutoxy aluminium hydride was added. The mixture was stirred for $1 \mathrm{~h}$, then diluted with ether $(5 \mathrm{~mL})$ and $0.5 \mathrm{M} \mathrm{H}_{3} \mathrm{PO}_{4}$-solution $(5 \mathrm{~mL})$ was added. The mixture was allowed to warm to room temperature. Phases were separated and the aqueous phase was extracted with ether. The combined organic extracts were washed with brine, dried over anhydrous sodium sulfate and evaporated to dryness. The crude product was purified by flash chromatography using 10-20\% ethyl acetate in hexanes to give in elution order fraction A of alcohol $16(0.028 \mathrm{~g}, 62 \%)$ as light yellow crystals and fraction B $0.016 \mathrm{~g}(35 \%)$ of a mixture of 16 and 17 as white solids. Total yield was 0.044 g (97\%). Selectivity was found to be syn:anti 3:1 based on crude NMR data. Alcohol 17 can be purified by another flash chromatography followed by recrystallization from ethyl acetate hexanes. Alternatively by chancing the reductant to L-Selectride, alcohol 17 can be obtained in 1:2 syn:anti-ratio.

For 16: $R_{f}=0.25$ (20\% EtOAc / hexanes); IR (thin film, $\mathrm{cm}^{-1}$ ) 3436, 2959, 2869, 1687, 1504, 1454, $1366,1247,1170 ;[\alpha]^{20}=-203.8\left(c 1.0, \mathrm{CHCl}_{3}{ }^{1} \mathrm{H}-\mathrm{NMR}\left(400 \mathrm{MHz}, \mathrm{CDCl}_{3}\right) \delta 5.80(\mathrm{~d}, J=9.6 \mathrm{~Hz}, 1 \mathrm{H})\right.$, $5.46(\mathrm{dt}, J=6.3,15.1 \mathrm{~Hz}, 1 \mathrm{H}), 5.39(\mathrm{ddd}, J=2.5,4.2,9.5 \mathrm{~Hz}, 1 \mathrm{H}), 5.28(\mathrm{dd}, J=9.0,15.2 \mathrm{~Hz}, 1 \mathrm{H}), 4.65$ (br s, 1H), 3.61 (br s, 1H), 3.38-3.49 (m, 1H), 2.79-2.91 (m, 1H), $2.01[\mathrm{dq}, J=7.4 \mathrm{~Hz}(\mathrm{~d}), 2 \mathrm{H}], 1.62-$ $1.92(\mathrm{~m}, 6 \mathrm{H}), 1.50-1.62(\mathrm{~m}, 2 \mathrm{H}), 1.20-1.49$ (obs m, 4H), 1.44 (obs s, 9H), 1.07-1.20 (obs m, 2H), 1.15 (obs d, $J=6.7 \mathrm{~Hz}, 3 \mathrm{H}), 0.97(\mathrm{t}, J=7.4 \mathrm{~Hz}, 3 \mathrm{H}) ;{ }^{13} \mathrm{C}-\mathrm{NMR}\left(100 \mathrm{MHz}, \mathrm{CDCl}_{3}\right) \delta$ 156.1, 133.5, 131.0, 129.4, 128.6, 79.3, 75.1, 50.4, 46.2, 44.7, 43.1, 42.4, 31.1, 29.3, 28.3, 27.9, 26.3, 25.6, 22.1, 18.3, 13.9; HRMS (ESI) calc. for $[\mathrm{M}+\mathrm{Na}] \mathrm{C}_{23} \mathrm{H}_{39} \mathrm{NO}_{3} \mathrm{Na} 400.2828$, found 400.2833, $\Delta=1.2 \mathrm{ppm}$. These data match those obtained from the orginal spectra. 
For 17: $R_{f}=0.20\left(20 \%\right.$ EtOAc / hexanes); IR (thin film, $\left.\mathrm{cm}^{-1}\right) 3365,2955,2868,1683,1527,1453$, 1367, 1176, 1042; $[\alpha]^{20}=-217.9\left(c 0.7, \mathrm{CHCl}_{3}\right) ;{ }^{1} \mathrm{H}-\mathrm{NMR}\left(400 \mathrm{MHz}, \mathrm{CDCl}_{3}\right) \delta 5.81(\mathrm{~d}, J=9.7 \mathrm{~Hz}$ 1H), $5.46(\mathrm{dt}, J=6.3,15.1 \mathrm{~Hz}, 1 \mathrm{H}), 5.39(\mathrm{ddd}, J=2.6,4.4,9.6 \mathrm{~Hz}, 1 \mathrm{H}), 5.26(\mathrm{dd}, J=9.1,15.1 \mathrm{~Hz}, 1 \mathrm{H})$, 4.77 (br s, 1H), 3.67 (br s, 1H), 3.52-3.61 (m, 1H), 2.82-2.92 (m, 1H), 2.05-2.28 (m, 1H), 1.95-2.05 (m, 2H), 1.63-1.88 (m, 5H), 1.50-1.63 (m, 3H), $1.44(\mathrm{~s}, 9 \mathrm{H}), 1.09-1.35(\mathrm{~m}, 5 \mathrm{H}), 1.05(\mathrm{~d}, J=6.8 \mathrm{~Hz}, 3 \mathrm{H})$, $0.96(\mathrm{t}, J=7.4 \mathrm{~Hz}, 3 \mathrm{H}) ;{ }^{13} \mathrm{C}-\mathrm{NMR}\left(100 \mathrm{MHz}, \mathrm{CDCl}_{3}\right) \delta 155.8,133.4,131.1,129.3,128.6,79.4,75.0$ 50.3, 46.2, 44.7, 43.3, 43.1, 30.8, 29.3, 28.4, 27.9, 27.3, 25.6, 22.1, 14.3, 13.9; HRMS (ESI) calc. for $[\mathrm{M}+\mathrm{Na}] \mathrm{C}_{23} \mathrm{H}_{39} \mathrm{NO}_{3} \mathrm{Na} 400.28,28$ found $400.2824, \Delta=1.0 \mathrm{ppm}$.
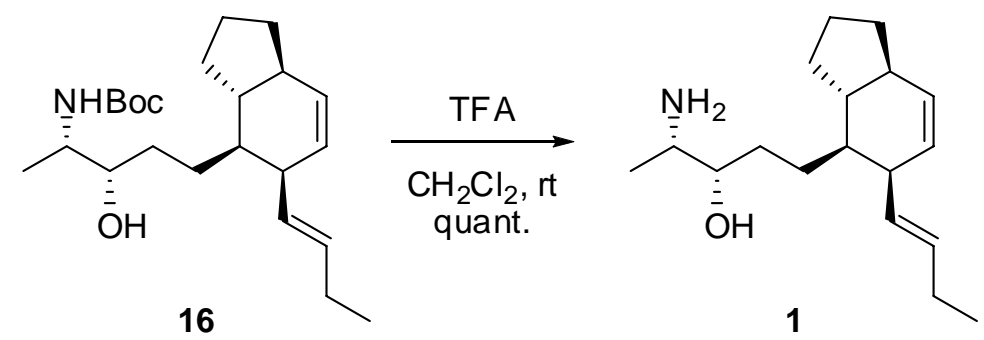

Amaminol A (1). Alcohol 16 (0.017 g, $0.045 \mathrm{mmol}, 100 \mathrm{~mol} \%)$ was dissolved in dichloromethane $(1 \mathrm{~mL})$ and trifluoroacetic acid $(0.1 \mathrm{~mL})$ was added. After $3 \mathrm{~h}$ the mixture was diluted with dichloromethane $(2 \mathrm{~mL})$ and $1 \mathrm{M} \mathrm{NaOH}(2 \mathrm{~mL})$ was added. The organic phase was washed with $1 \mathrm{M} \mathrm{NaOH}(2 \mathrm{~mL})$. The combined aqueous phases were extracted with dichloromethane $(2 \mathrm{~mL}) . \mathrm{The}$ combined organic extracts were dried over anhydrous sodium sulfate and evaporated to give amaminol A $1\left(0.013 \mathrm{~g}\right.$, quant.) as an oil. IR (thin film, $\left.\mathrm{cm}^{-1}\right)$ 3262, 2958, 2870, 1739, 1601, 1454, 1077, 974; $[\alpha]^{20}=-214.8\left(c 0.7, \mathrm{CHCl}_{3}\right) ;{ }^{1} \mathrm{H}-\mathrm{NMR}(400 \mathrm{MHz}, d 4-\mathrm{MeOD}) \delta 5.79(\mathrm{~d}, J=9.87 \mathrm{~Hz}, 1 \mathrm{H}), 5.48(\mathrm{dt}, J=$ 5.9, $15.7 \mathrm{~Hz}, 1 \mathrm{H}), 5.29-5.43(\mathrm{~m}, 2 \mathrm{H}), 3.16-3.27(\mathrm{~m}, 1 \mathrm{H}), 2.84-2.93(\mathrm{~m}, 1 \mathrm{H}), 2.69(\mathrm{dq}, J=6.5 \mathrm{~Hz}(\mathrm{~d})$, 1H), $2.03[\mathrm{ddq}, J=1.1,7.4 \mathrm{~Hz}(\mathrm{~d}), 2 \mathrm{H}], 1.66-1.88(\mathrm{~m}, 5 \mathrm{H}), 1.56-1.66(\mathrm{~m}, 1 \mathrm{H}), 1.34-1.55(\mathrm{~m}, 4 \mathrm{H}), 1.10-$ $1.25(\mathrm{~m}, 2 \mathrm{H}), 1.04(\mathrm{~d}, J=6.4 \mathrm{~Hz}, 3 \mathrm{H}), 0.98(\mathrm{t}, J=7.4 \mathrm{~Hz}, 3 \mathrm{H}) ;{ }^{13} \mathrm{C}-\mathrm{NMR}(100 \mathrm{MHz}, d 4-\mathrm{MeOD}) \delta$ $134.7,132.2,130.1,130.0,77.2,52.1,47.7,46.0,44.2,44.0,31.7,30.4,28.9,27.3,26.7,23.1,19.3$ 14.3; HRMS (ESI) calc. for $[\mathrm{M}+\mathrm{H}] \mathrm{C}_{18} \mathrm{H}_{32} \mathrm{NO} 278.2484$, found 278.2479, $\Delta=1.8 \mathrm{ppm}$. 
Amaminol A 1 was converted to its corresponding TFA salt for comparison with the original spectral data. For 1·TFA: $[\alpha]^{20}=-88.3(c 1.0, \mathrm{MeOH}) ;{ }^{1} \mathrm{H}-\mathrm{NMR}(400 \mathrm{MHz}, d 4-\mathrm{MeOD}) \delta 5.80(\mathrm{~d}, J=9.7 \mathrm{~Hz}$, $1 \mathrm{H}), 5.50(\mathrm{dt}, J=6.2,14.9 \mathrm{~Hz}, 1 \mathrm{H}), 5.29-5.43(\mathrm{~m}, 2 \mathrm{H}), 3.46(\mathrm{dt}, J=2.9,7.6 \mathrm{~Hz}, 1 \mathrm{H}), 3.09(\mathrm{dq}, J=6.6$, $6.8 \mathrm{~Hz}, 1 \mathrm{H}), 2.85-2.94(\mathrm{~m}, 1 \mathrm{H}), 1.98-2.08(\mathrm{~m}, 2 \mathrm{H}), 1.67-1.89(\mathrm{~m}, 5 \mathrm{H}), 1.36-1.66(\mathrm{~m}, 5 \mathrm{H}), 1.25(\mathrm{~d}, J=$ $6.7 \mathrm{~Hz}, 3 \mathrm{H}), 1.10-1.23(\mathrm{~m}, 2 \mathrm{H}), 0.98(\mathrm{t}, J=7.2 \mathrm{~Hz}) ;{ }^{13} \mathrm{C}-\mathrm{NMR}(100 \mathrm{MHz}, d 4-\mathrm{MeOD}) \delta$ 134.8, 132.0, $130.2,129.9,73.1,53.1,47.6,45.9,44.2,44.0,31.8,30.4,28.9,26.8,26.7,23.1,15.9,14.3$. These data match those reported in the literature. ${ }^{6}$
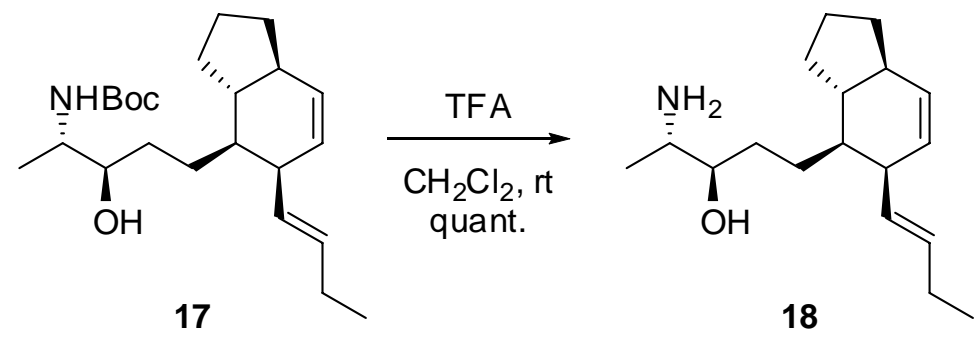

(2S,3R)-epi-Amaminol A (18). The compound was prepared as described for 1 starting from 17 (7 mg, $18 \mu \mathrm{mol}, 100 \mathrm{~mol} \%$ ) giving 18 (5 mg, quant.) as an oil. IR (thin film, $\mathrm{cm}^{-1}$ ) 3330, 2956, 2869, 1729, 1695, 1454, 1375, 1297,1181, 998, 969; $[\alpha]^{20}=-213.4\left(c\right.$ 0.5, $\left.\mathrm{CHCl}_{3}\right) ;{ }^{1} \mathrm{H}-\mathrm{NMR}(400 \mathrm{MHz}$, $\left.\mathrm{CDCl}_{3}\right) \delta 5.79(\mathrm{~d}, J=9.6 \mathrm{~Hz}, 1 \mathrm{H}), 5.50(\mathrm{dt}, J=6.3,15.2 \mathrm{~Hz}, 1 \mathrm{H}), 5.38(\mathrm{ddd}, J=2.5,4.4,9.7 \mathrm{~Hz}, 1 \mathrm{H})$, 5.30 (ddt, $J=1.3,9.0,15.1 \mathrm{~Hz}, 1 \mathrm{H}), 3.33-3.94(\mathrm{~m}, 1 \mathrm{H}), 2.86-2.96(\mathrm{~m}, 1 \mathrm{H}), 2.73-2.83(\mathrm{~m}, 1 \mathrm{H}), 2.02$ $[\mathrm{ddq}, J=1.2,7.4 \mathrm{~Hz}(\mathrm{~d}), 2 \mathrm{H}], 1.50-1.89(\mathrm{~m}, 8 \mathrm{H}), 1.09-1.39(\mathrm{~m}, 5 \mathrm{H}), 1.02(\mathrm{~d}, J=6.6 \mathrm{~Hz}, 3 \mathrm{H}), 0.97(\mathrm{t}, J$ $=7.5 \mathrm{~Hz}, 3 \mathrm{H}) ;{ }^{13} \mathrm{C}-\mathrm{NMR}\left(100 \mathrm{MHz}, \mathrm{CDCl}_{3}\right) \delta 134.5,132.2,130.1(\mathrm{~d}), 77.0,51.8,47.6,46.1,44.6,31.2$, 30.4, 29.0, 28.8, 26.7, 23.1, 16.8, 14.4; HRMS (ESI) calc. for $[\mathrm{M}+\mathrm{H}] \mathrm{C}_{18} \mathrm{H}_{32} \mathrm{NO} 278.2484$, found 278.2495, $\Delta=4.0 \mathrm{ppm}$. 

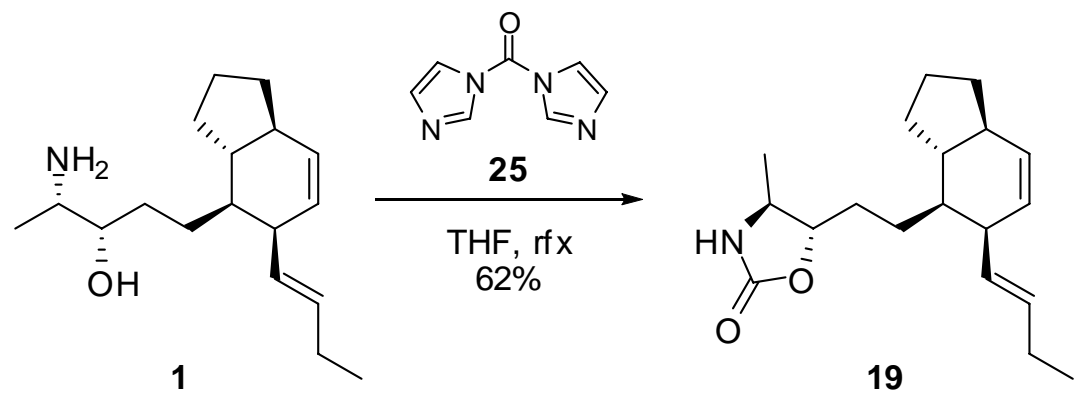

\section{(4S,5S)-5-(2-((3aR,4S,5R,7aS)-5-((E)-but-1-enyl)-2,3,3a,4,5,7a-hexahydro-1H-inden-4-yl)ethyl)-}

4-methyloxazolidin-2-one (19). To a solution of aminoalcohol 1 (7 mg, $26 \mu \mathrm{mol}, 100 \mathrm{~mol} \%)$ in dry THF (2 mL) under argon was added carbonyl di-imidazole (6.4 mg, $40 \mu \mathrm{mol}, 150 \mathrm{~mol} \%)$. The mixture was refluxed for $19 \mathrm{~h}$. The solvent was evaporated and the crude product was purified by flash chromatography using 50\% ethyl acetate in hexanes as eluent to give of carbamate $19(5 \mathrm{mg}, 62 \%)$ as white crystals. Needle shape crystals for x-ray crystallography were obtained by recrystallization from EtOAc $(30 \mu \mathrm{L}) /$ hexanes $(0.3 \mathrm{~mL}) . R_{f}=0.50\left(\right.$ EtOAc); IR (thin film, $\mathrm{cm}^{-1}$ ) 3256, 2956, 2927, 2868, $1752,1454,1393,1239 ;[\alpha]^{20}=-190.2\left(c 0.5, \mathrm{CHCl}_{3}\right) ;{ }^{1} \mathrm{H}-\mathrm{NMR}\left(400 \mathrm{MHz}, \mathrm{CDCl}_{3}\right) \delta 5.82(\mathrm{~d}, J=9.6$ $\mathrm{Hz}, 1 \mathrm{H}), 5.46(\mathrm{dt}, J=6.3,15.2 \mathrm{~Hz}, 1 \mathrm{H}), 5.40(\mathrm{ddd}, J=2.6,4.2,9.6 \mathrm{~Hz}, 1 \mathrm{H}), 5.18-5.30(\mathrm{~m}, 2 \mathrm{H}), 4.08$ [ddd, $J=4.8,6.8 \mathrm{~Hz}(\mathrm{~d}), 1 \mathrm{H}$ ], 3.57 [dq, $J=6.3 \mathrm{~Hz}(\mathrm{~d}), 1 \mathrm{H}$ ], 2.80-2.90 (m, 1H), 2.01 [ddq, $J=1.1,7.3$ $\mathrm{Hz}(\mathrm{d}), 2 \mathrm{H}], 1.76-1.88(\mathrm{~m}, 3 \mathrm{H}), 1.64-1.76(\mathrm{~m}, 3 \mathrm{H}), 1.52-1.63(\mathrm{~m}, 2 \mathrm{H}), 1.31-1.52(\mathrm{~m}, 3 \mathrm{H}), 1.26(\mathrm{~d}, J=$ $6.0 \mathrm{~Hz}, 3 \mathrm{H}), 1.06-1.21(\mathrm{~m}, 2 \mathrm{H}), 0.96(\mathrm{t}, J=7.4 \mathrm{~Hz}, 3 \mathrm{H}) ;{ }^{13} \mathrm{C}-\mathrm{NMR}\left(100 \mathrm{MHz}, \mathrm{CDCl}_{3}\right) \delta 158.8,133.8$, $130.8,129.4,128.2,84.3,53.2,46.2,44.6,43.2,42.2,30.9,29.9,27.9,25.7,25.6,22.1,20.6,14.0$; HRMS (ESI) calc. for [M+Na] $\mathrm{C}_{19} \mathrm{H}_{29} \mathrm{NO}_{2} \mathrm{Na} 326.2096$, found 326.2089, $\Delta=2.1 \mathrm{ppm}$. These data match those obtained from the original spectra. 

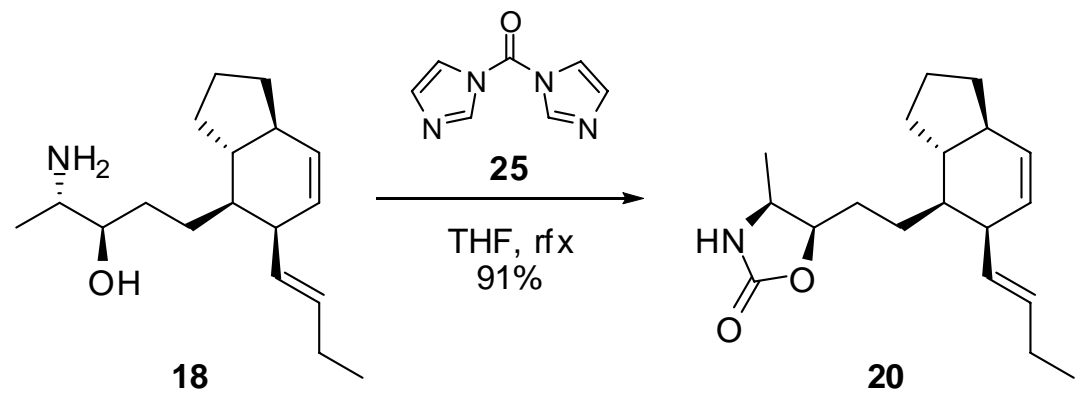

(4S,5R)-5-(2-((3aR,4S,5R,7aS)-5-((E)-but-1-enyl)-2,3,3a,4,5,7a-hexahydro-1H-inden-4-yl)ethyl)-

4-methyloxazolidin-2-one (20). The compound was prepared as described for $\mathbf{1 9}$ starting from 18 (9 $\mathrm{mg}, 32 \mu \mathrm{mol})$ giving $\mathbf{2 0}(9 \mathrm{mg}, 91 \%)$ as a clear oil. $R_{f}=0.42$ (EtOAc); IR (thin film, $\left.\mathrm{cm}^{-1}\right) 3270,2955$, 2926, 2869, 1751, 1455, 1389, 1232, 1107, 970; $[\alpha]^{20}=-111.8\left(c 0.8, \mathrm{CHCl}_{3}\right) ;{ }^{1} \mathrm{H}-\mathrm{NMR}(400 \mathrm{MHz}$, $\left.\mathrm{CDCl}_{3}\right) \delta 5.81(\mathrm{~d}, J=9.7 \mathrm{~Hz}, 1 \mathrm{H}), 5.46(\mathrm{dt}, J=6.2,15.2 \mathrm{~Hz}, 1 \mathrm{H}), 5.39(\mathrm{ddd}, J=2.5,4.4,9.7 \mathrm{~Hz}, 1 \mathrm{H})$, 5.22-5.32 (m, 2H), 4.41-4.51 (m, 1H), $3.85(\mathrm{dq}, J=6.5,6.7 \mathrm{~Hz}, 1 \mathrm{H}), 2.83-2.91(\mathrm{~m}, 1 \mathrm{H}), 1.96-2.05(\mathrm{~m}$, 2H), 1.46-1.88 (m, 10H), 1.17-1.36 (m, 2H), 1.05-1.19 (obs m, 1H), 1.15 (obs d, $J=6.6 \mathrm{~Hz}, 3 \mathrm{H}$ ), 0.95 $(\mathrm{t}, J=7.4 \mathrm{~Hz}, 3 \mathrm{H}) ;{ }^{13} \mathrm{C}-\mathrm{NMR}\left(100 \mathrm{MHz}, \mathrm{CDCl}_{3}\right) \delta 159.3,133.6,130.8,129.4,128.5,80.9,51.1,46.2$, 44.6, 43.3, 42.7, 29.2, 27.9, 27.3, 26.5, 25.6, 22.1, 16.0, 13.9; HRMS (ESI) calc. for [M+Na] $\mathrm{C}_{19} \mathrm{H}_{29} \mathrm{NO}_{2} \mathrm{Na} 326.2096$, found 326.2112, $\Delta=4.9 \mathrm{ppm}$.

Relative stereochemistry analysis. To verify the relative stereochemistries, the aminoalcohols $\mathbf{1}$ and 18 were converted to the cyclic carbamate analogues 19 and 20. Coupling constants between H2-H3 were and $6.3 \mathrm{~Hz}$ (for 19) and $6.7 \mathrm{~Hz}$ (for 20) and therefore we could not rely on this data for accurate determination of the relative stereochemistry. However, NOE experiments combined with molecular modeling obtained with Hyperchem software v. 7.51 with MM+ theory suggested the stereochemical assignments to be $2 S, 3 S$ for $\mathbf{1 9}$ and $2 S, 3 R$ for $\mathbf{2 0}$ (Figure 1). 


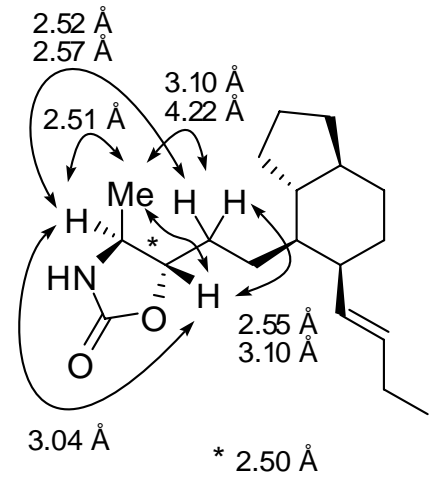

$(S, S)$-isomer 19

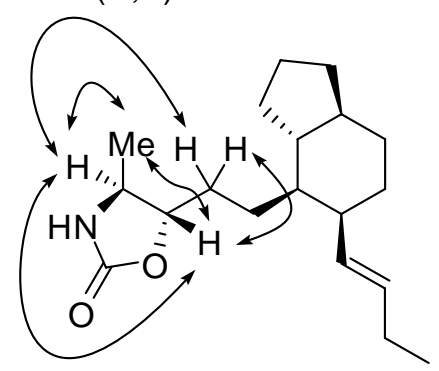

weak NOE

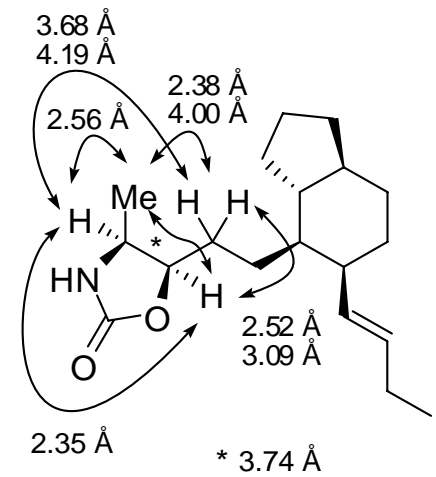

$(\mathrm{S}, \mathrm{R})$-isomer 20

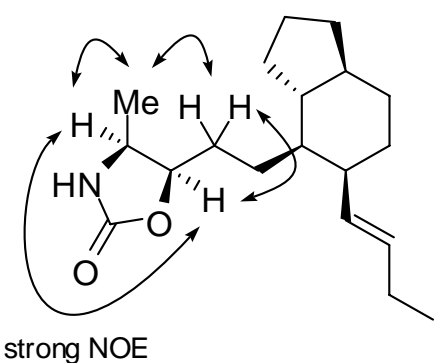

Figure 1. NOE-study of absolute stereochemistries of $\mathbf{1 9}$ and 20. Measured distances from molecular modeling are shown on top and found NOE's are presented at the bottom.

X-ray crystallography. The absolute stereochemistry was assigned based on the synthesis procedure and confirmed qualitatively by the weak anomalous dispersion effects during the refinements. Crystal data for 19 (Figure 2): formula $\mathrm{C}_{19} \mathrm{H}_{29} \mathrm{NO}_{2}$, crystal size $0.2 \times 0.3 \times 0.3 \mathrm{~mm}$, monoclinic, space group $P 2_{1}\left(\right.$ no. 4), $a=11.886(1) \AA, b=5.3996(4) \AA, c=14.772(1) \AA, \beta=104.433(4)^{\circ}, V=918.1(1) \AA^{3}, \mathrm{Z}=$ 2, $\rho_{\text {calcd }}=1.098 \mathrm{~cm}^{-3}, \mathrm{MoK}_{\alpha}$ radiation, $\lambda=0.71069 \AA, \mu=0.070 \mathrm{~mm}^{-1}, T=173.0(1) \mathrm{K}$, Bruker-Nonius Kappa CCD diffractometer, 16295 reflections measured, 3220 independent, 2597 with $I>2 \mathrm{~s}(I)(2.85<$ $\left.2 \theta<50^{\circ}\right), 201$ parameters, $R_{\mathrm{int}}=0.0369, R 1=0.0595[I>2 \mathrm{~s}(I)], w R 2=0.1451, \mathrm{GOF}=1.107$. Minimum and maximum peaks in the difference map -0.172 and $0.317 \mathrm{e}^{-3}$. Crystallographic data (excluding structure factors) for $\mathbf{1 9}$ have been deposited with the Cambridge Crystallographic Data Centre as supplementary publication no. 648016. Copies of the data can be obtained free of charge on application to CCDC, 12 Union Road Cambridge CB21EZ UK (fax: (+44)1223-336-033; e-mail: deposit@ccdc.cam.ac.uk). 


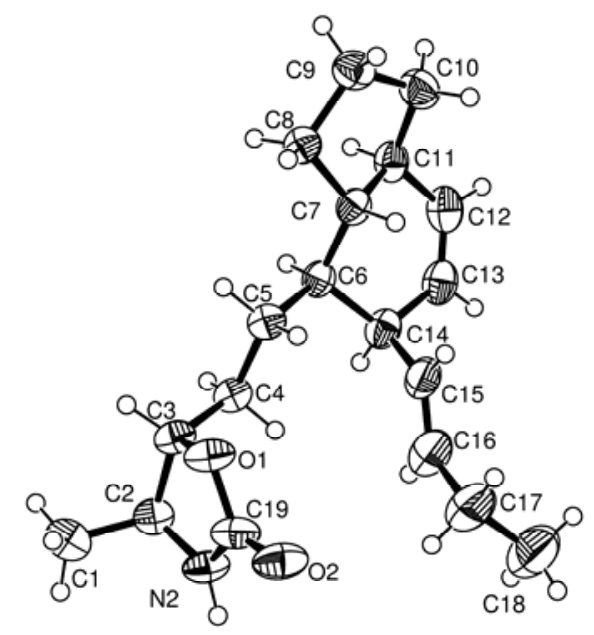

Figure 2. A plot of the X-ray crystal structure of carbamate 19 with atom labels and thermal displacement parameters at $50 \%$ probability level.

\section{References}

${ }^{1}$ This material is commercially available.

${ }^{2}$ Gunatilaka, A. A. L.; Hirai, N.; Kingston, D. G. I. Tetrahedron Lett. 1983, 24, 5457-5460. No NMR data available.

${ }^{3}$ a) S. L. Schreiber, R. E. Claus, J. Reagan, Tetrahedron Lett. 1982, 23, 3867-3870. b) J. M.

Aurrecoechea, J. H. Gil, B. López, Tetrahedron 2003, 59, 7111-7121.

${ }^{4}$ Little, R. D.; Fox, D. P.; Van Hijfte, L.; Dannecker, R.; Sowell, G.; Wolin, R. L.; Moens, L.; Baizer, M. M. J. Org. Chem. 1988, 53, 2287-2294.

${ }^{5}$ P. K. Chakravarty, W. J. Greenlee, W. H. Parsons, A. A. Patchett, P. Combs, A. Roth, R. D. Busch, T. N. Mellin, J. Med. Chem. 1989, 32, 1886-1890.

${ }^{6}$ N. U. Sata, N. Fusetani, Tetrahedron Lett. 2000, 41, 489-492. Original spectra for 1, 16, 19 were provided by professor Shigeki Matsunaga, University of Tokyo. 
Aldehyde 5 de and ee analysis
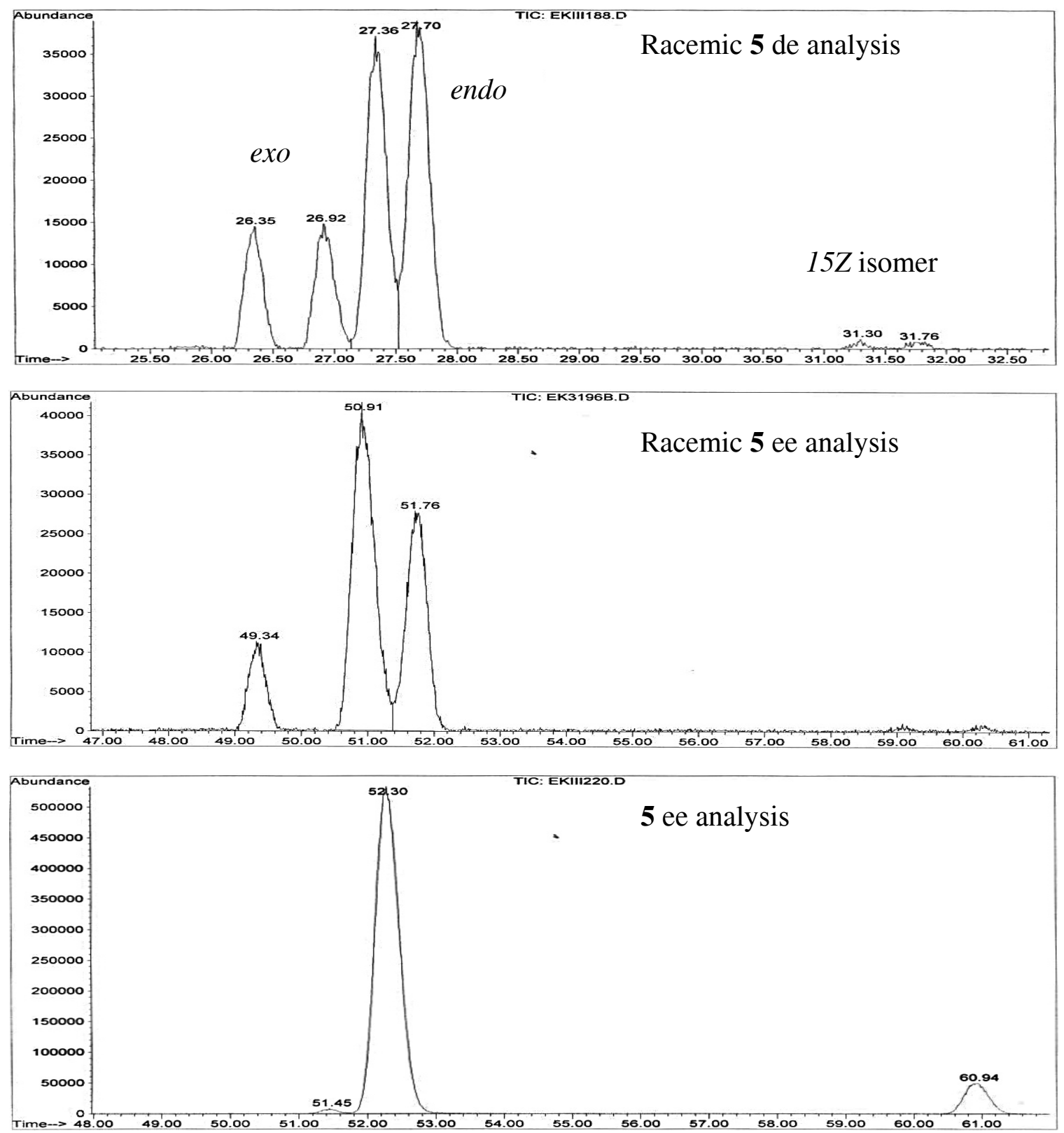

Data File : C: \HPCHEM \1\DATA \EKIII220.D

Acq on

: 25 Aug 2006 16:06

$\begin{array}{lll}\text { Sample } & \text { EKIII220 ee analysis } & \text { Inst : GC/MS } \\ \text { Misc } & : \text { inj270C,10psi, } 130 \mathrm{C}, 65 \mathrm{~m},-220 \mathrm{C}, 8 \mathrm{C} / \mathrm{m}, 30 \mathrm{~m} \text {, de Multiplr: } 1.00\end{array}$

Vial: 17

Sample

perator: EK

MS Integration Params: AUTOINT1.E

Sample Amount: 0.00
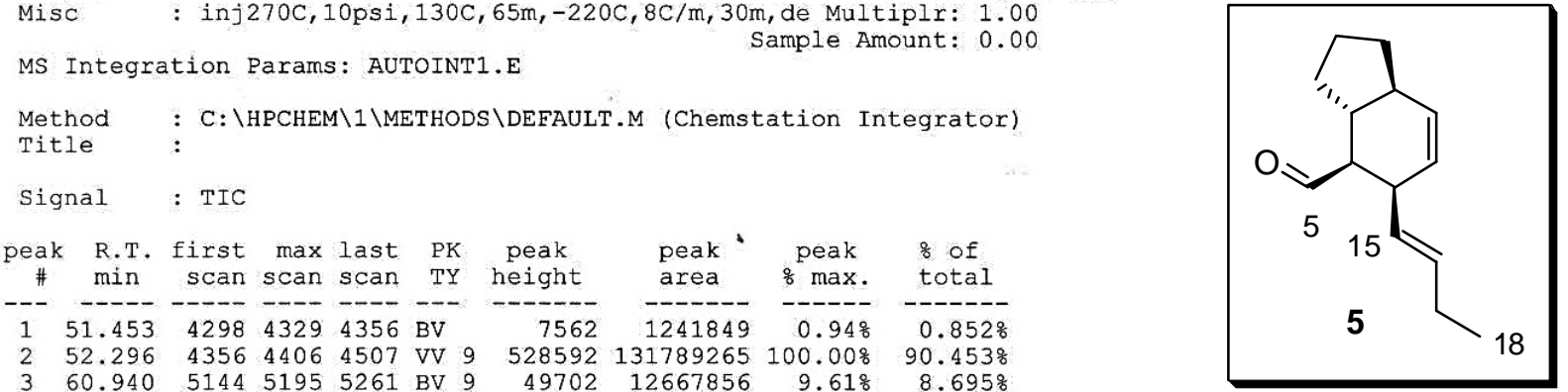

Sum of corrected areas: 145698970

EKIII220.D DEFAULT.M Mon Jan 08 17:14:51 2007 


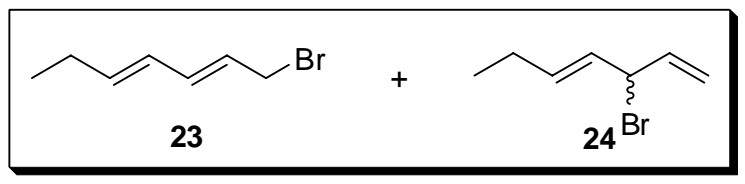

EKIV6 kugel dist.

20.11.2005 $\mathrm{CDCl}^{3}$

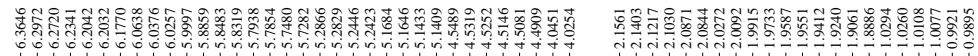 \\ (n)}

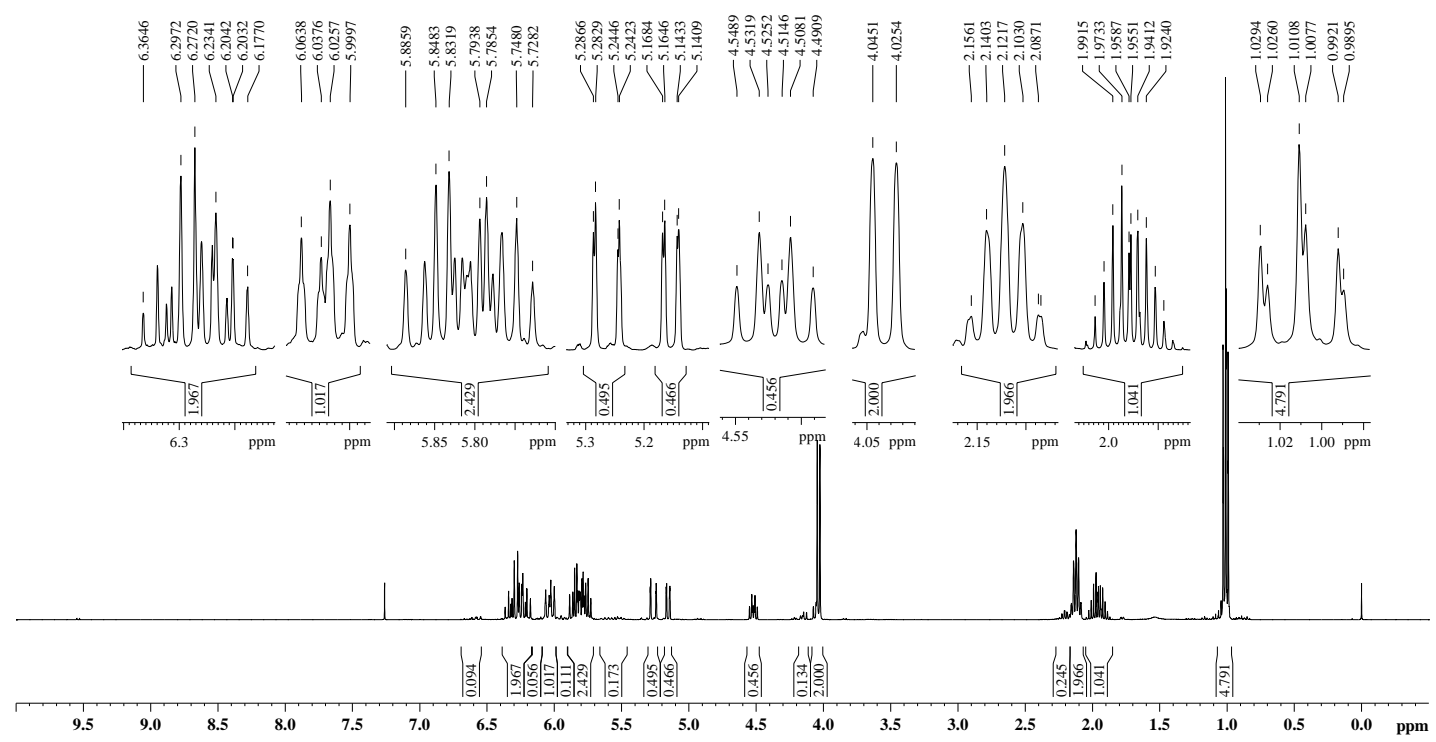

EKIV6 kugel dist

20.11.2005 CDCl3

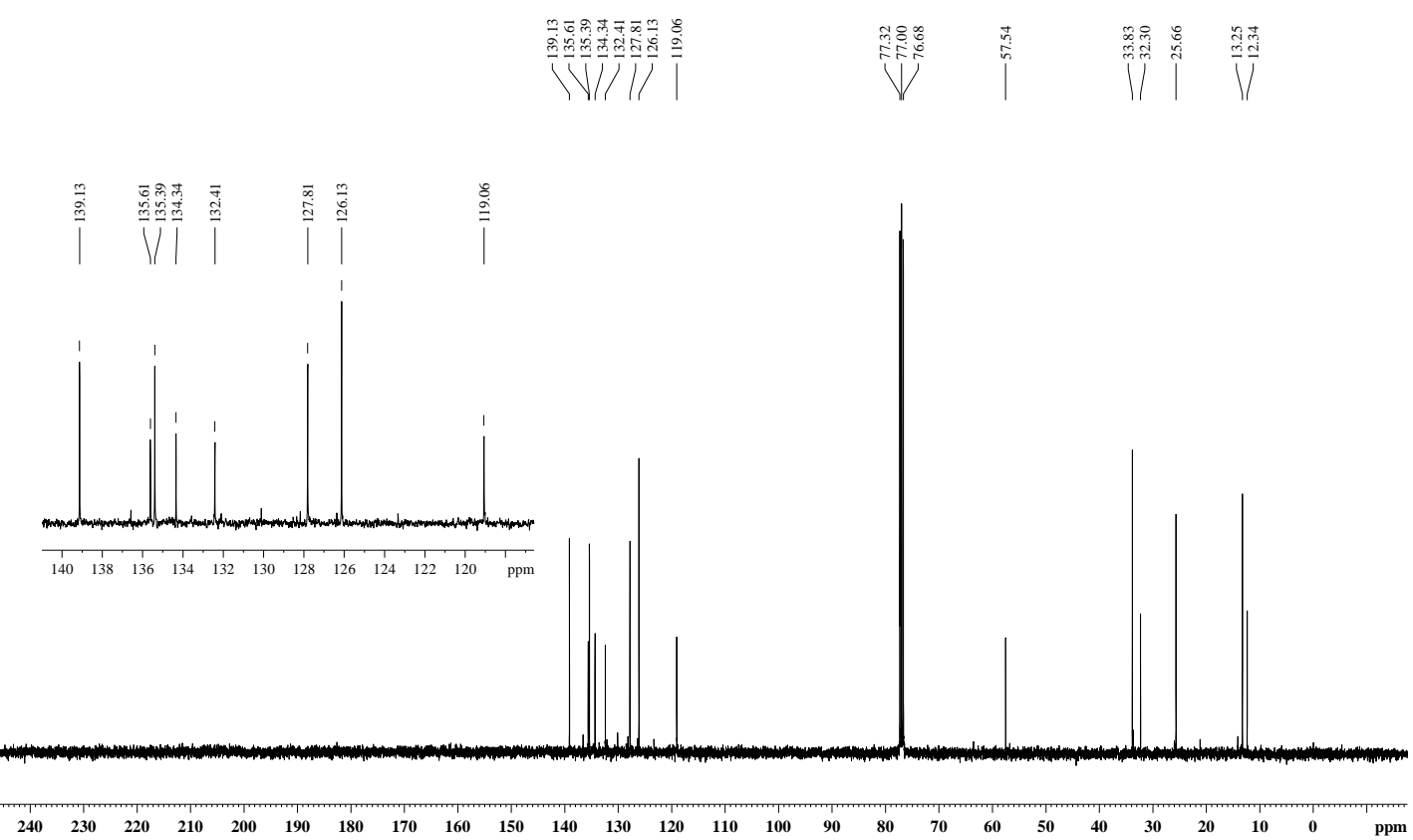




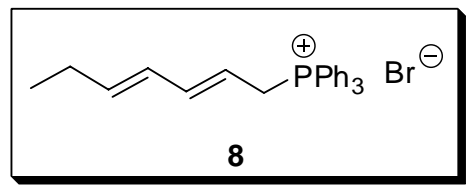

EKIII208 crude

19.07.2006 CDCl3

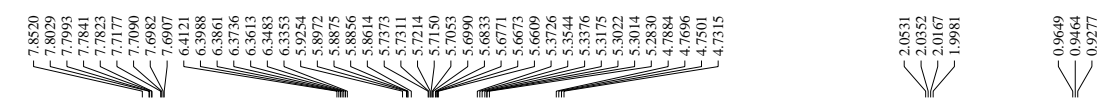

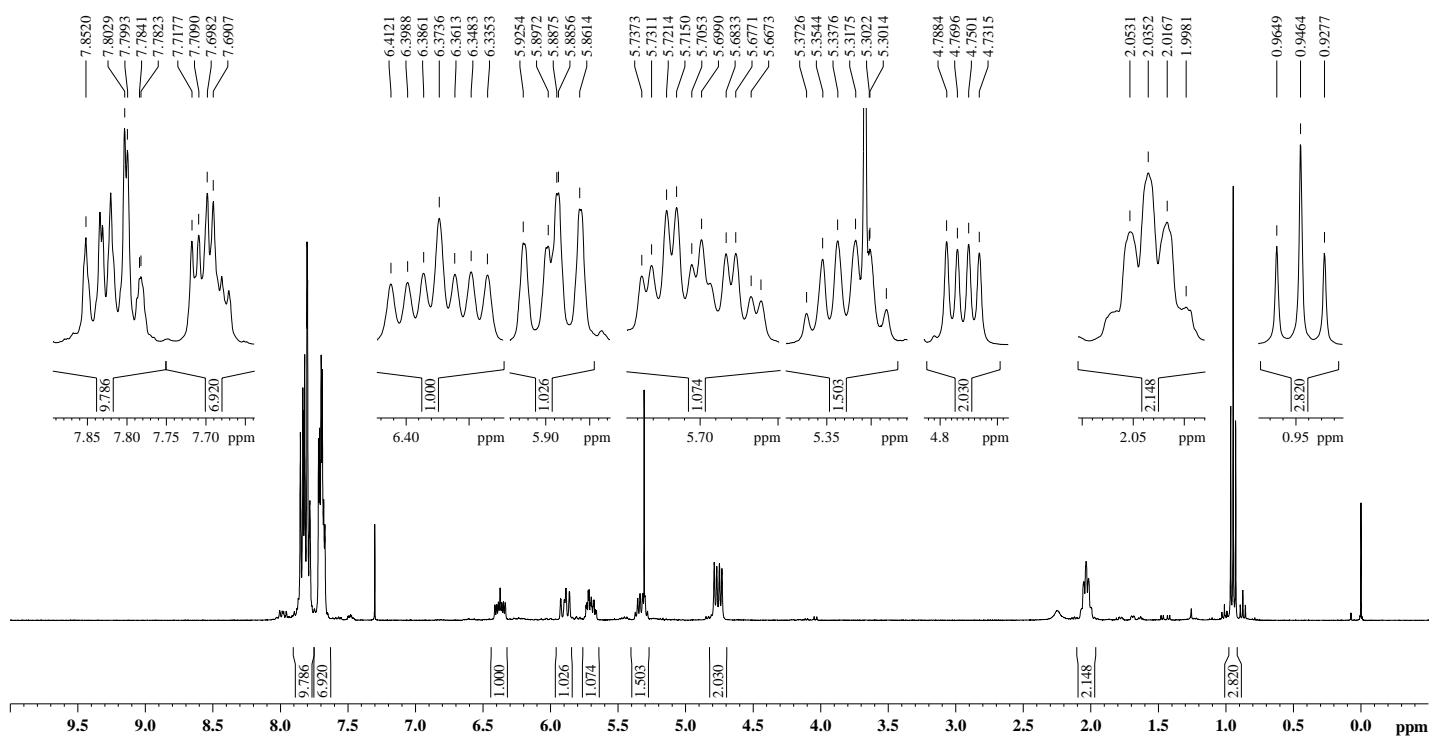

EKIII208 crude

19.07.2006 CDCl3
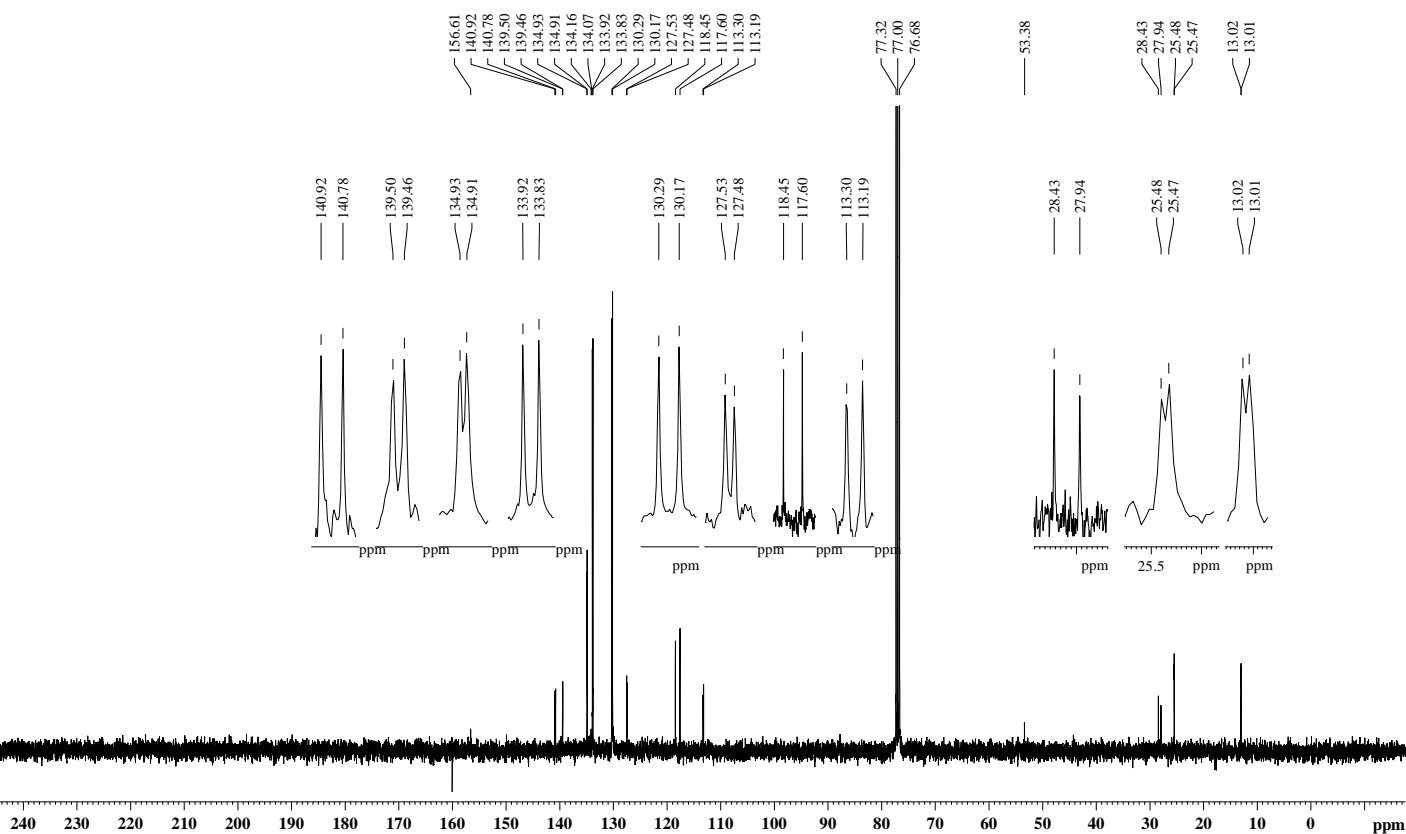


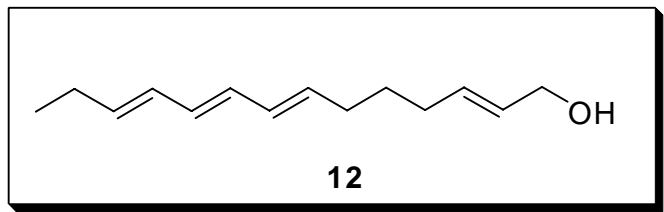

EKIII212 f3-7

18.08.2006 $\mathrm{CDCl} 3$

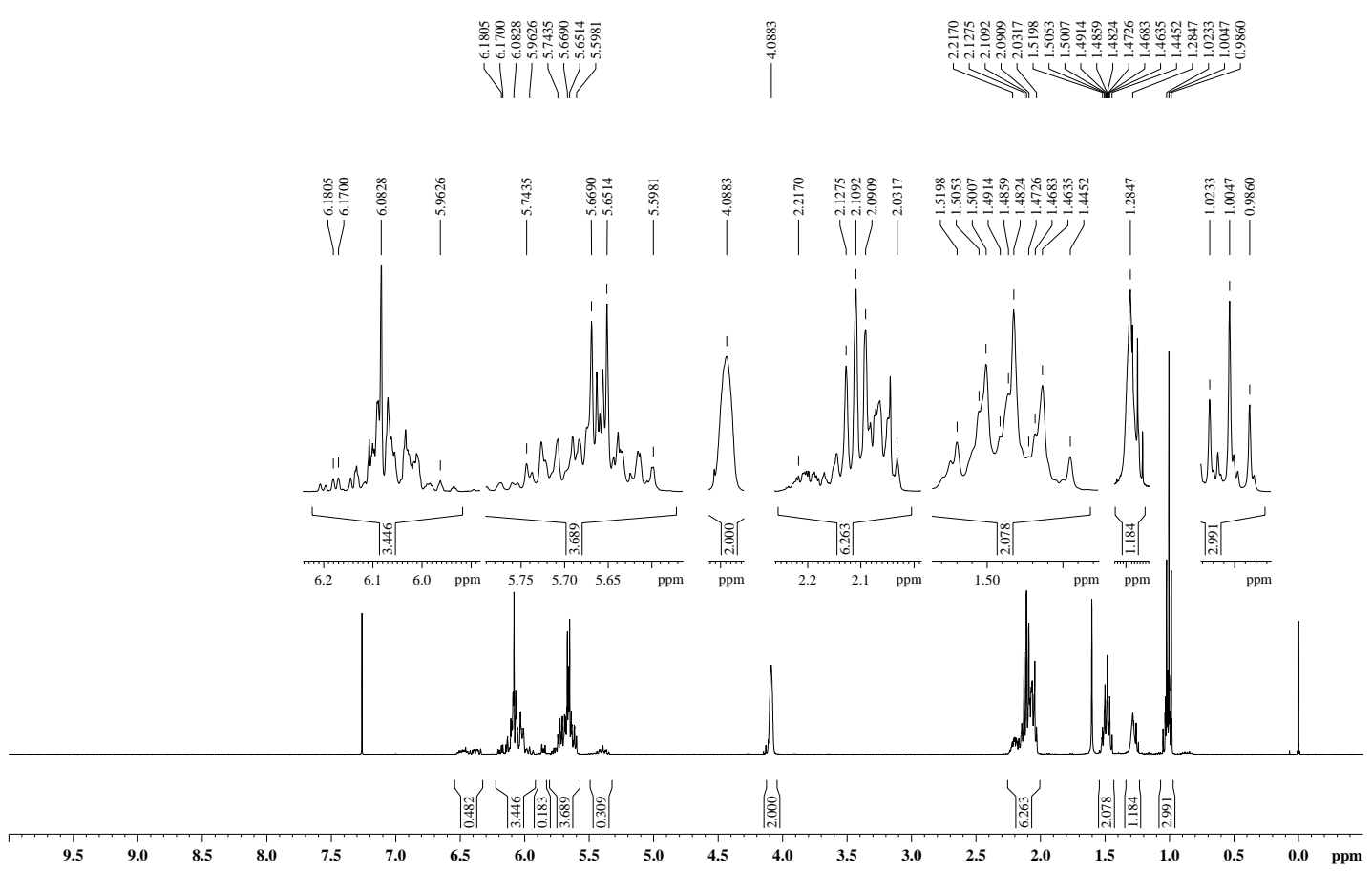

EKIII174 f6-13

17.05.2006 CDCl3

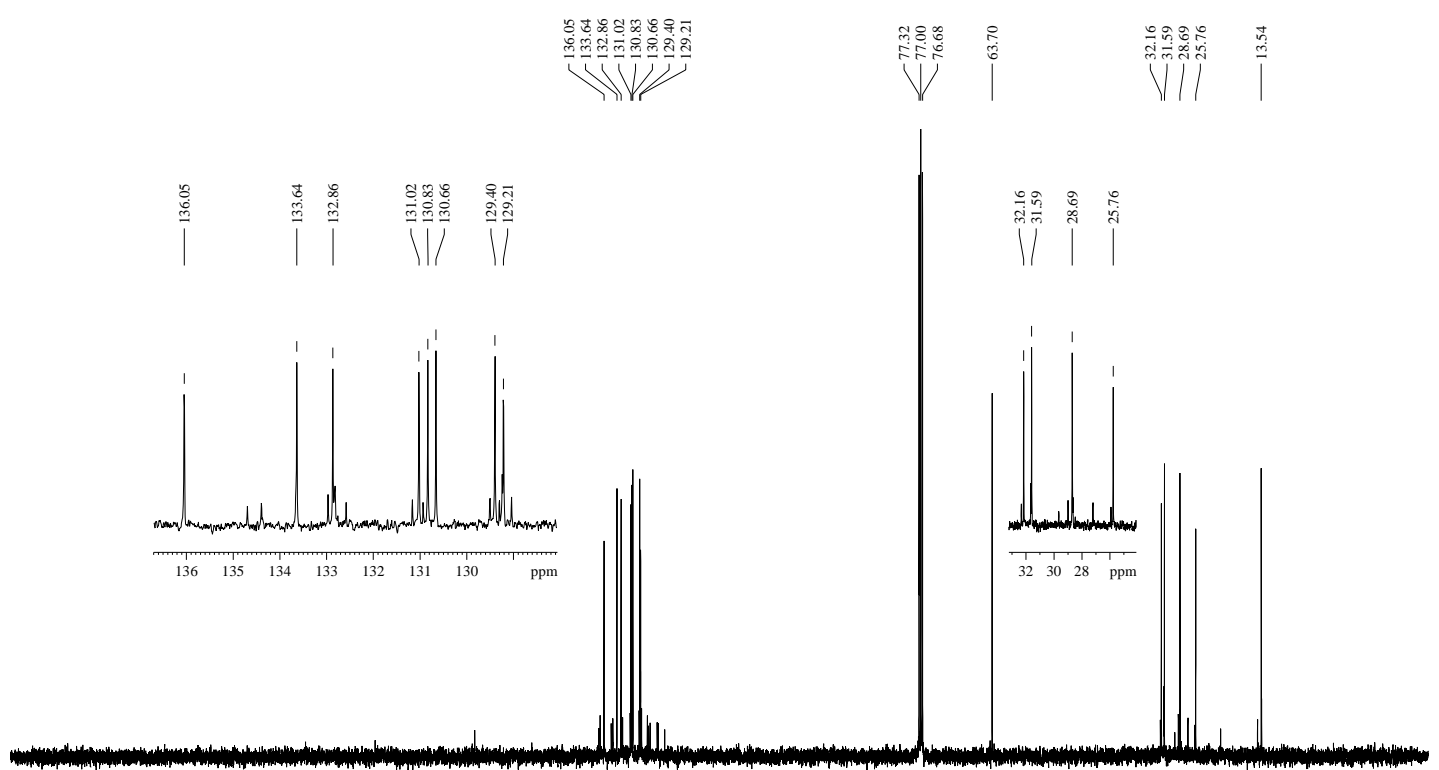

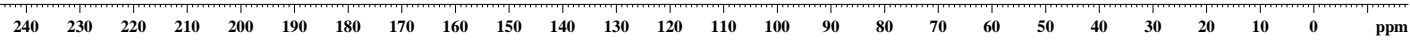




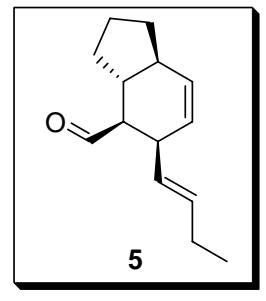

EKIII220 f8-15

25.08.2006 CDCl3

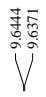

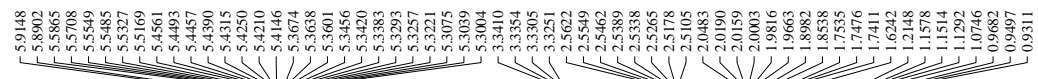

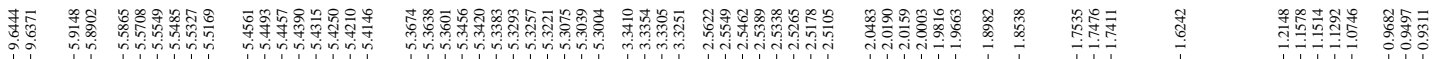

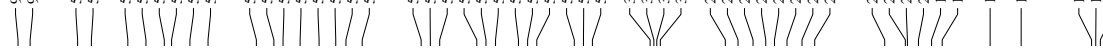
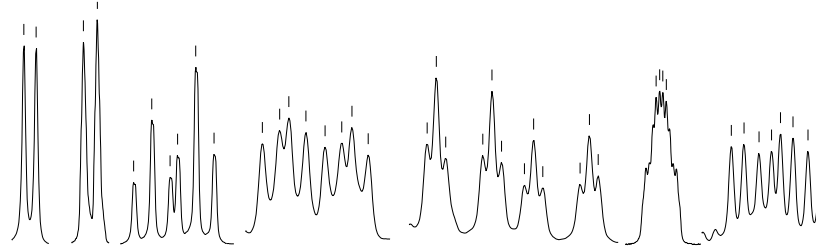

$\operatorname{Lim}_{m}^{1}$
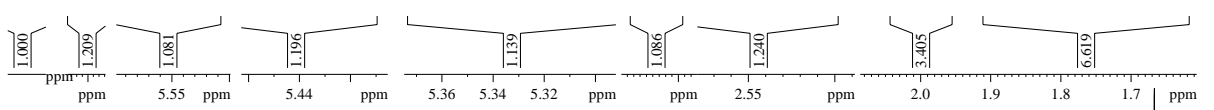

hindill
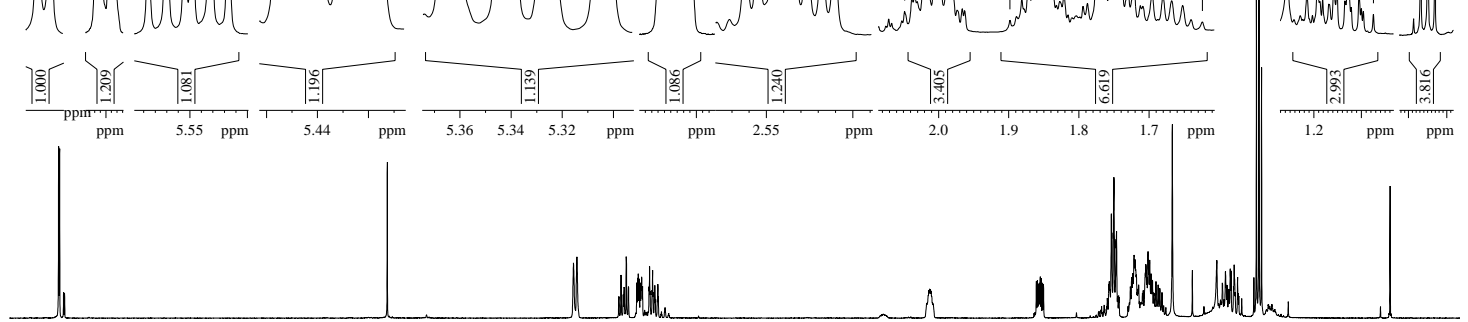

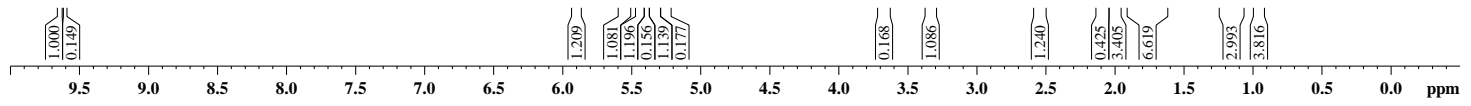

EKIII220 f8-15

25.08.2006 CDCl3

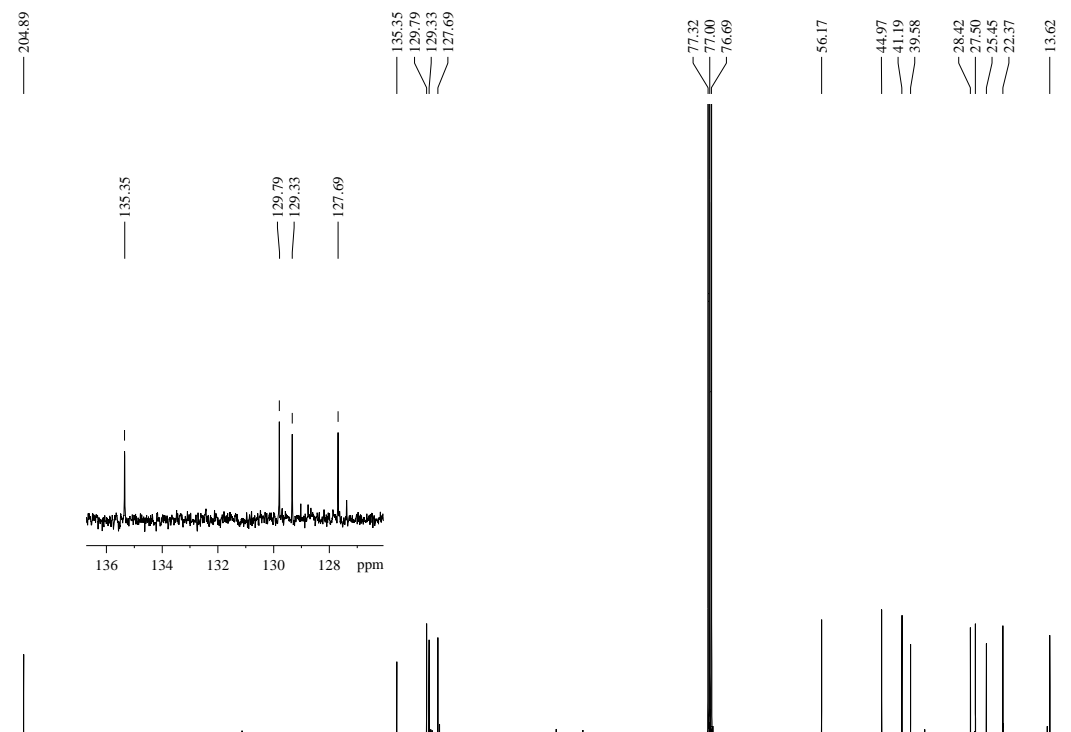

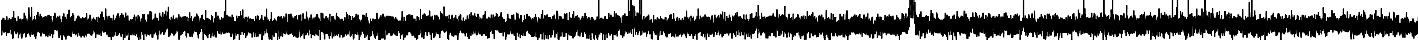

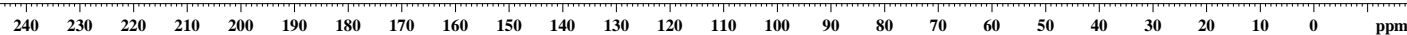




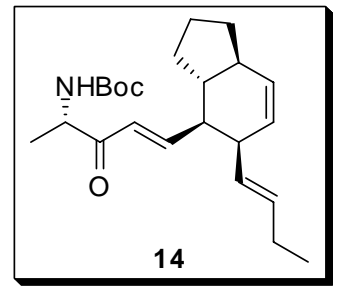

EKIII238 f7-16

22.09.2006 CDCl3

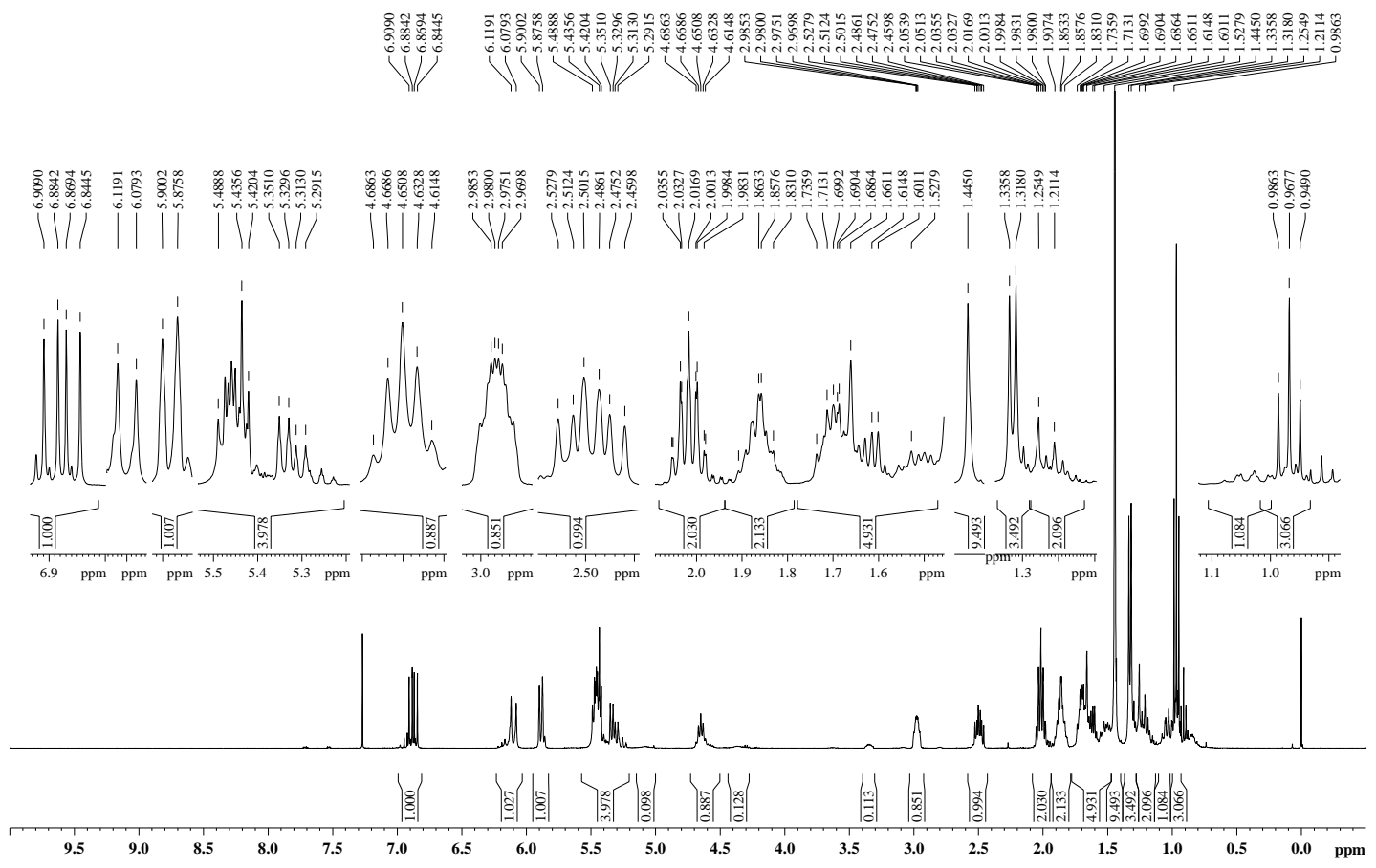

EKIII238 f7-16

22.09.2006 CDCl3

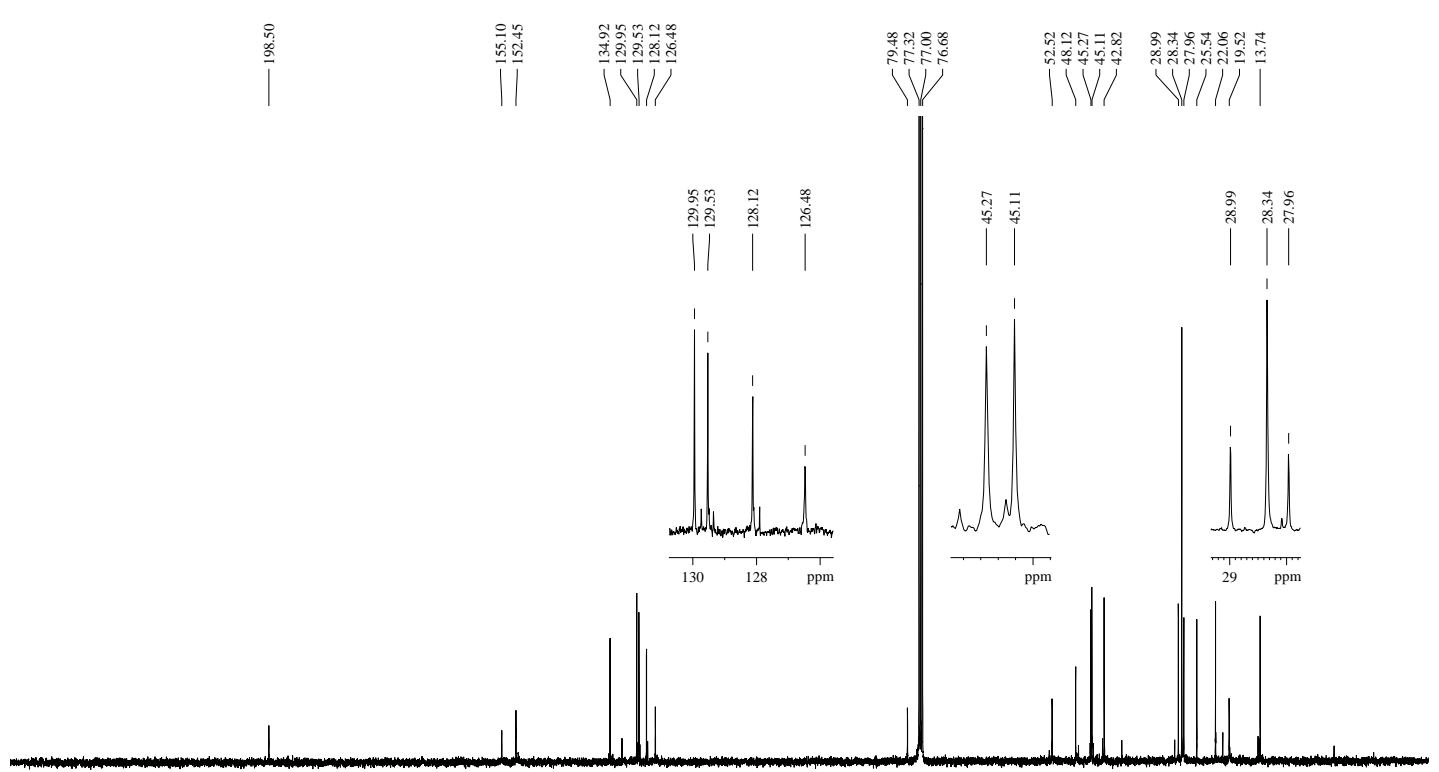

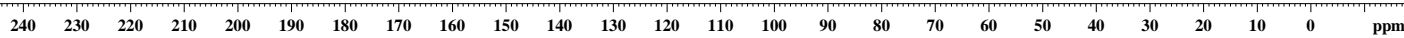




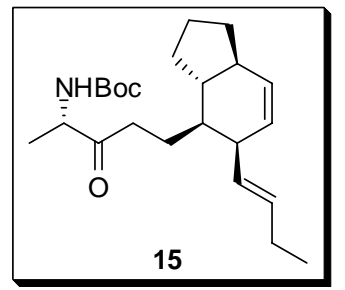

EKIV116 f11-37

18.05.2007 CDCl3

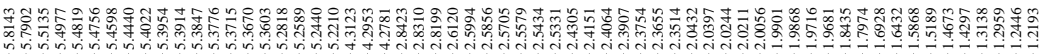
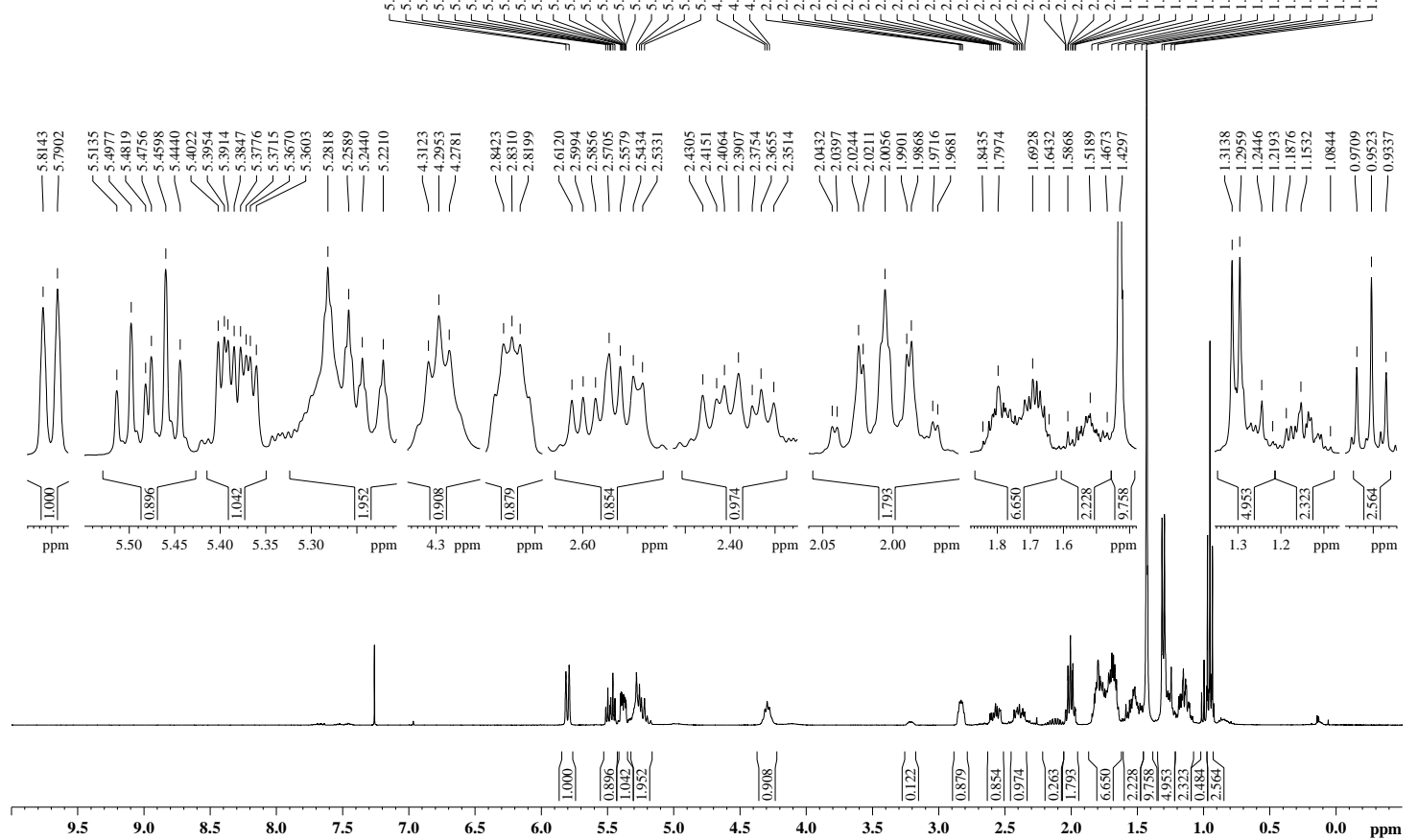

EKIV110 f9-26

05.05.2007 CDCl3

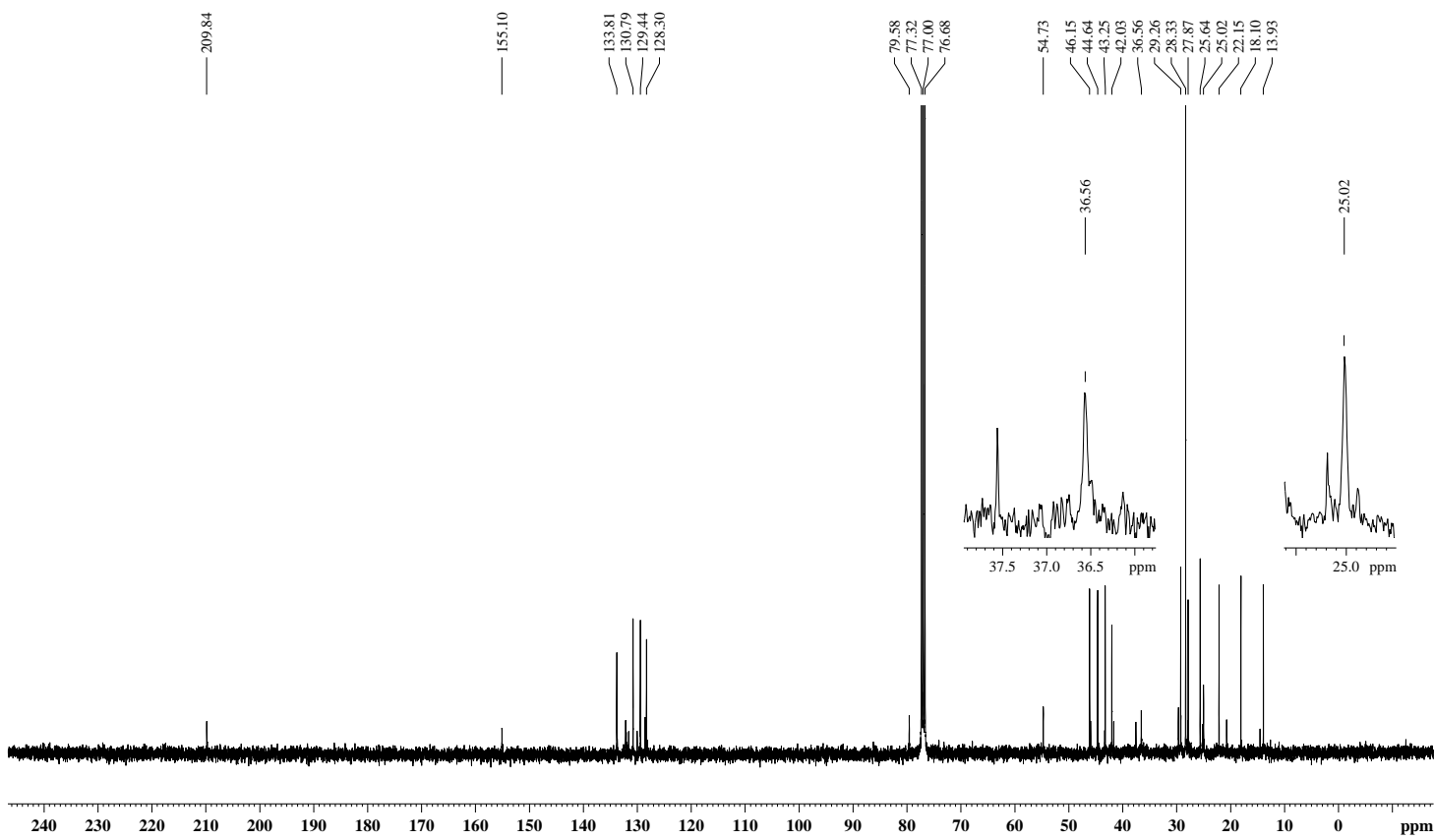




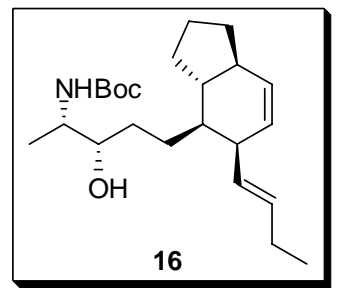

EKIV118 f26-41 recryst.

24.05.2007 CDCl3

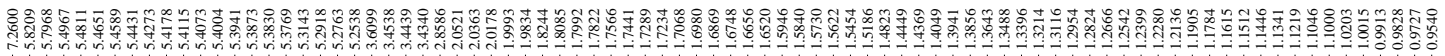
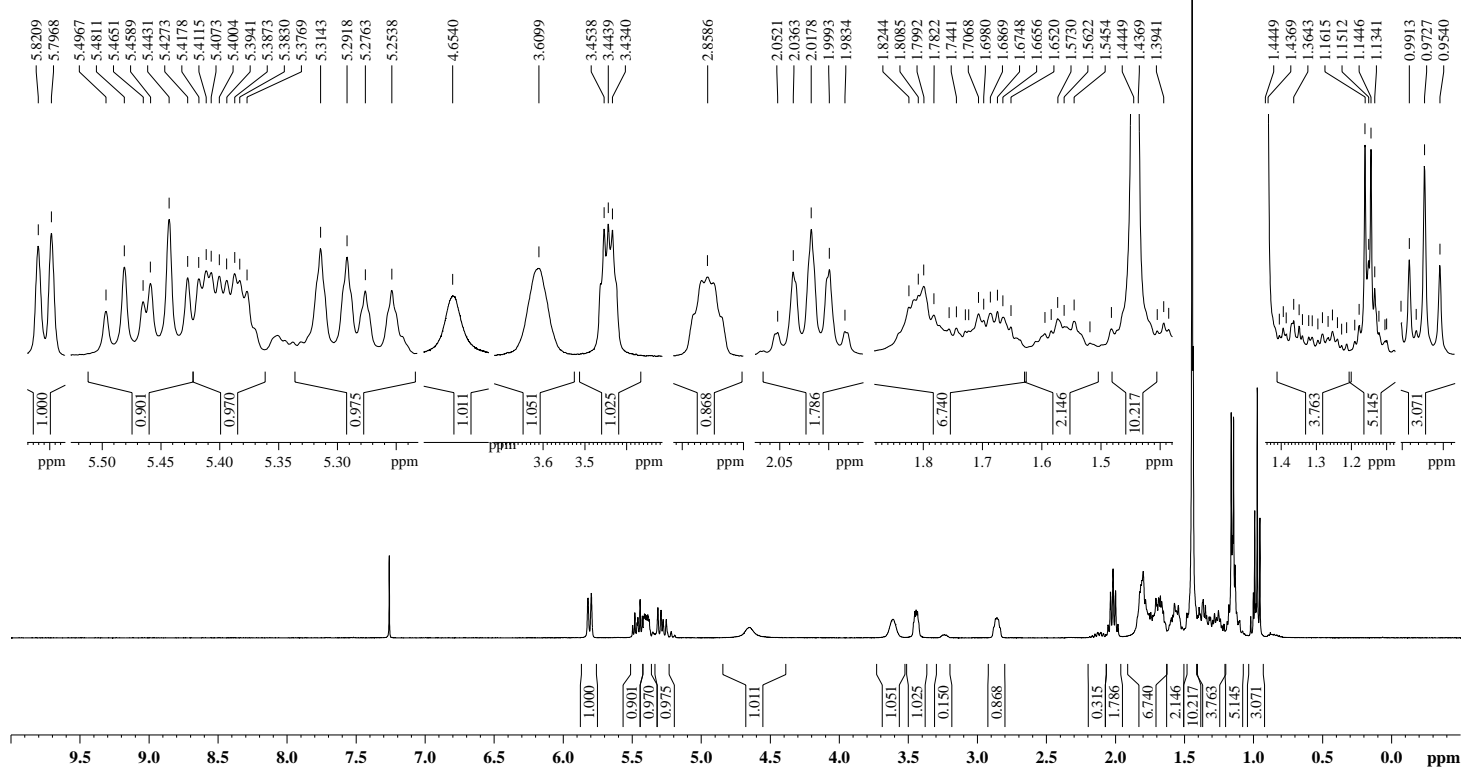

EKIV118 f26-41 recryst. 24.05.2007 CDCl3

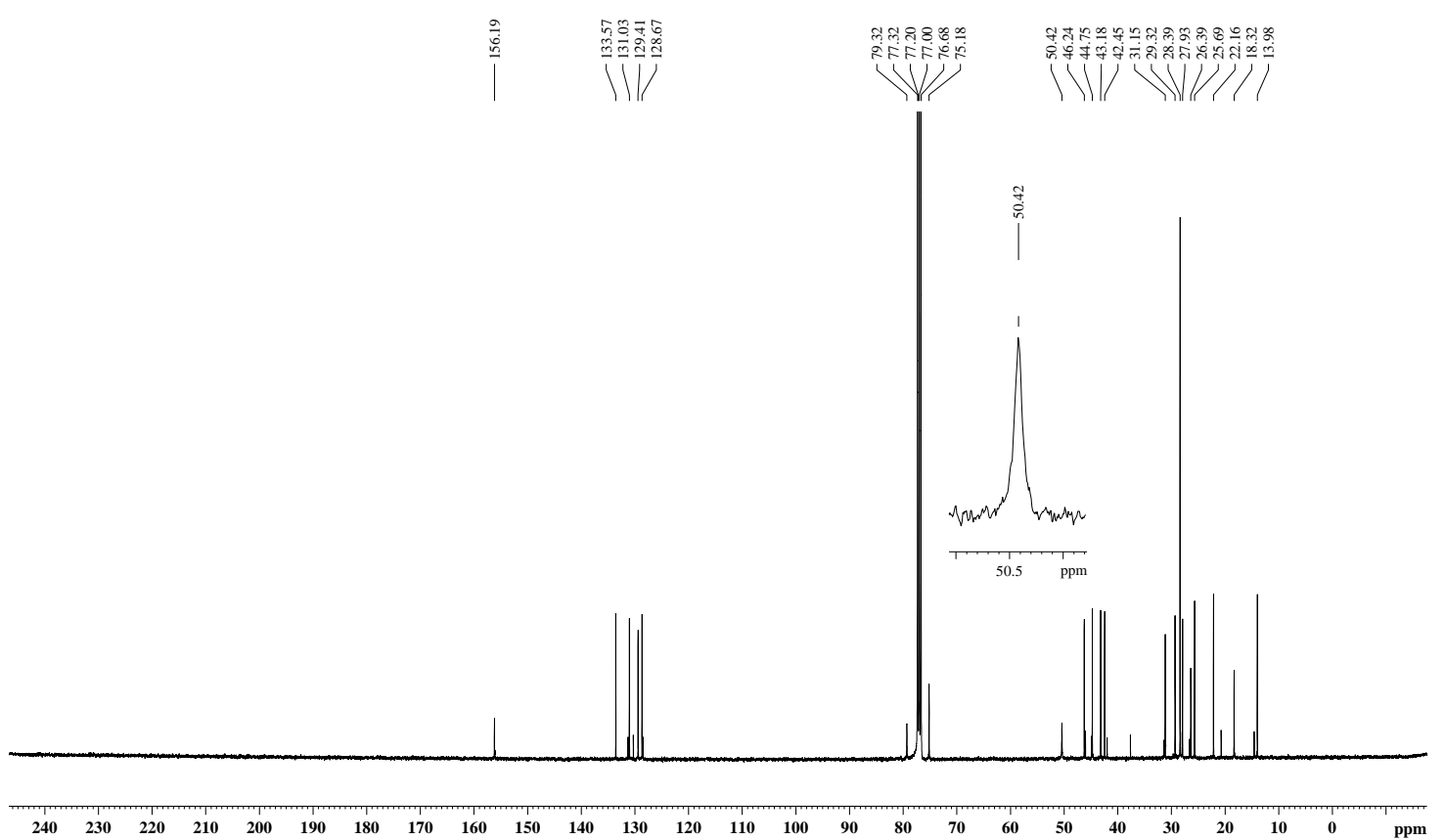




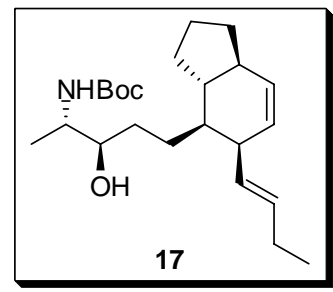

EKIV82B recryst.

26.03.2007 CDCl3

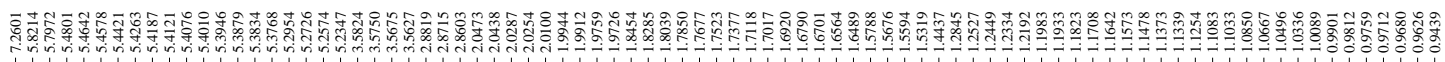

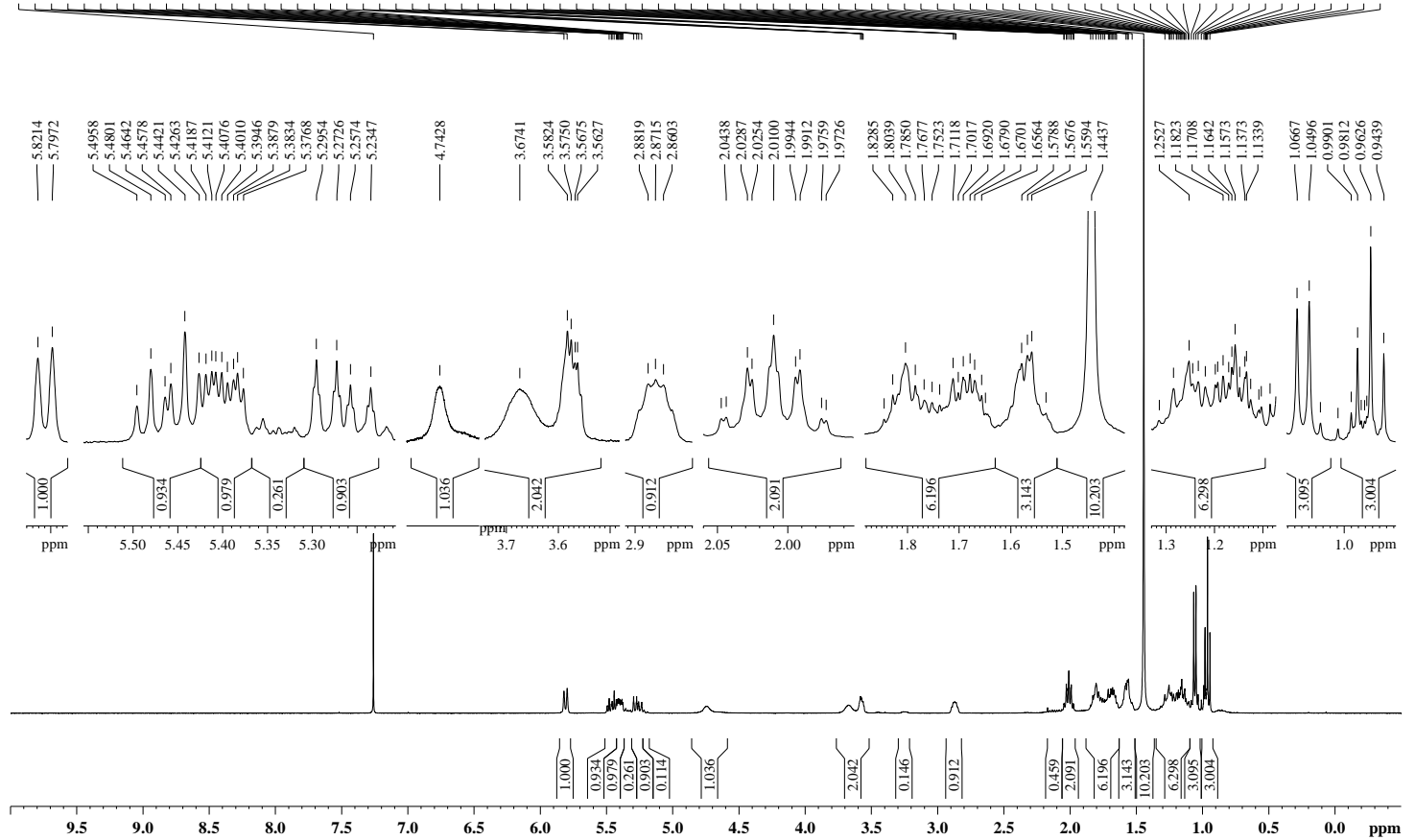

EKIV82B recryst.

05.05.2007 CDCl3

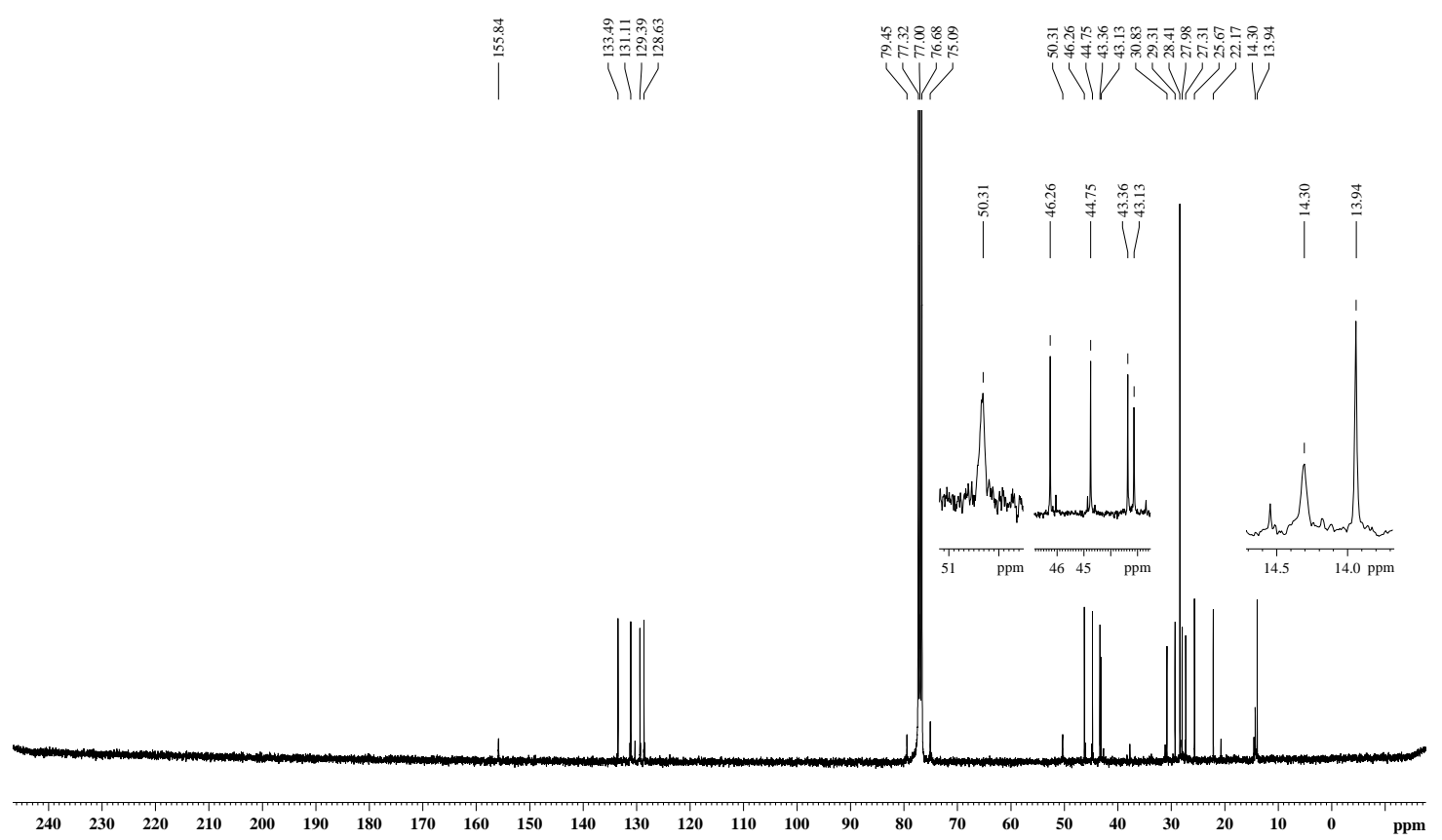




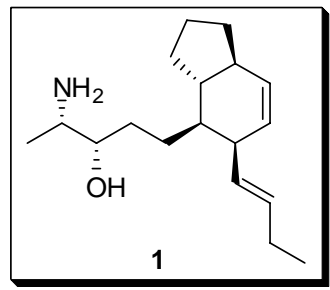

EKIV128 crude

30.05.2007 MeOD

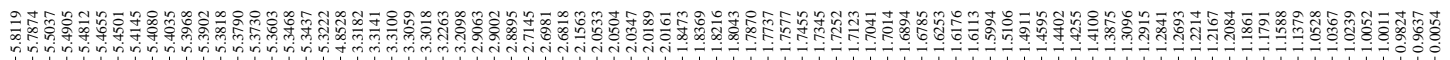

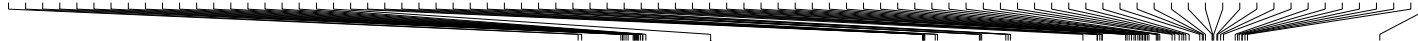

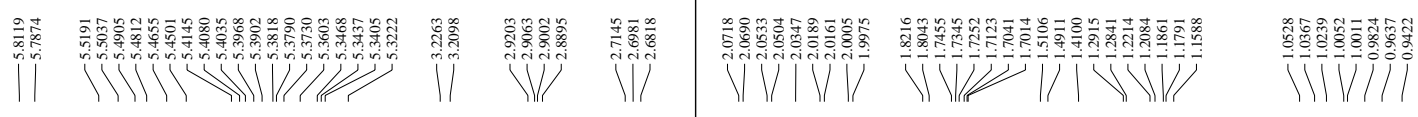
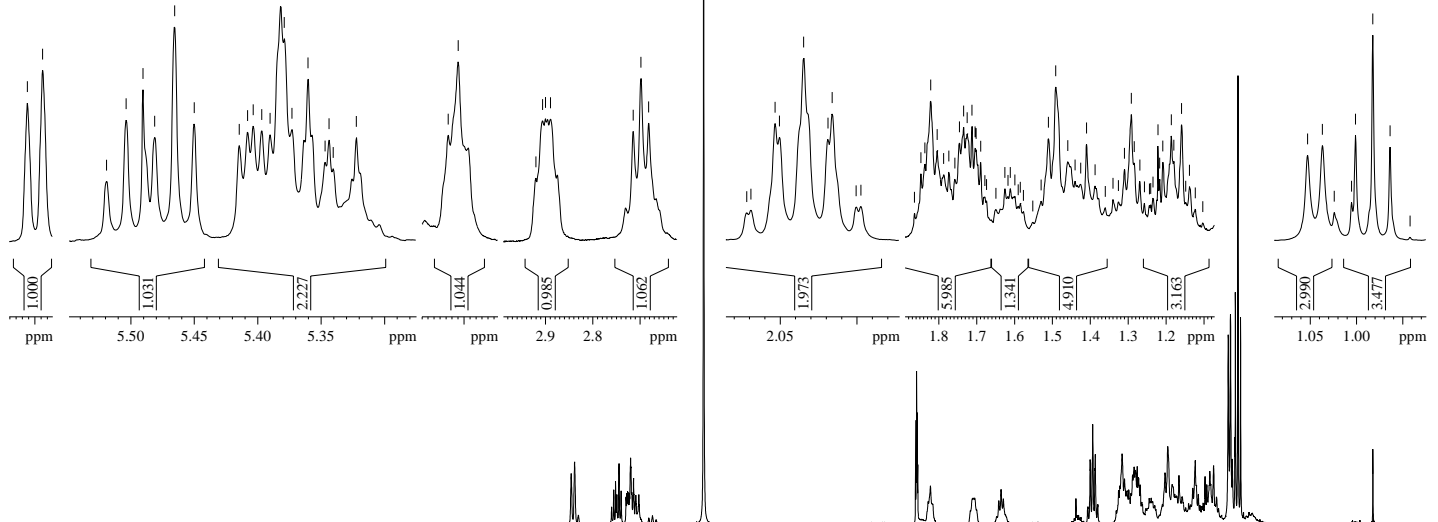

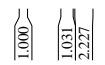

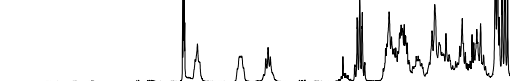

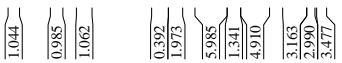

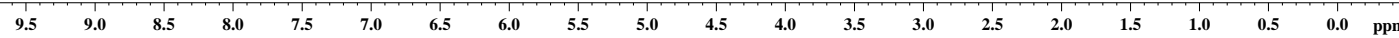

EKIV128 crude

30.05.2007 MeOD

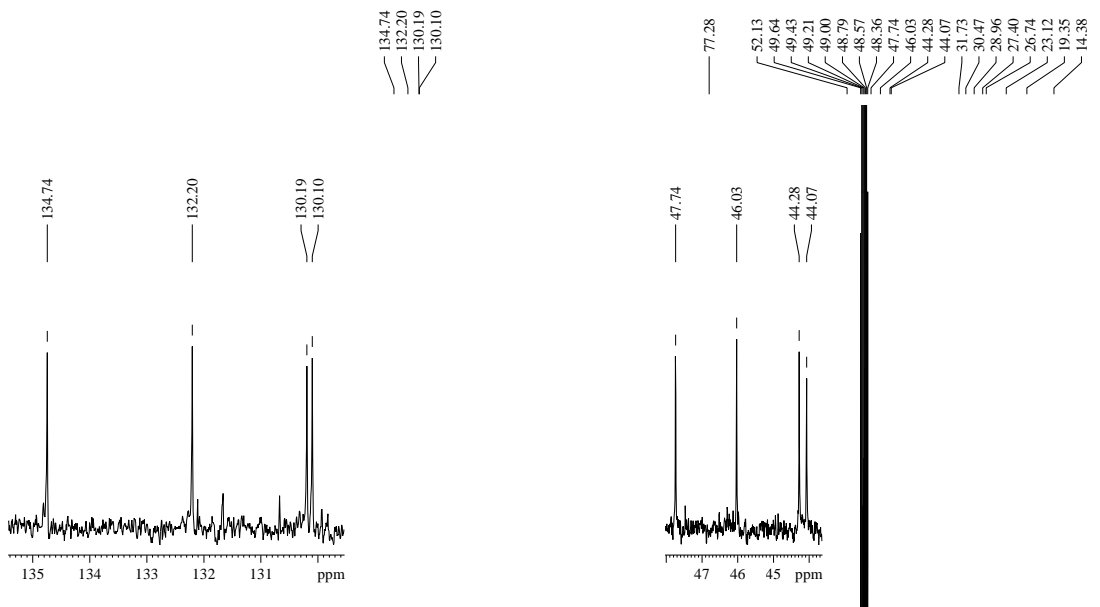




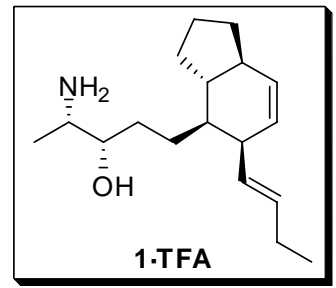

EKIV128 rescued + TFA

07.06.2007 MeOD

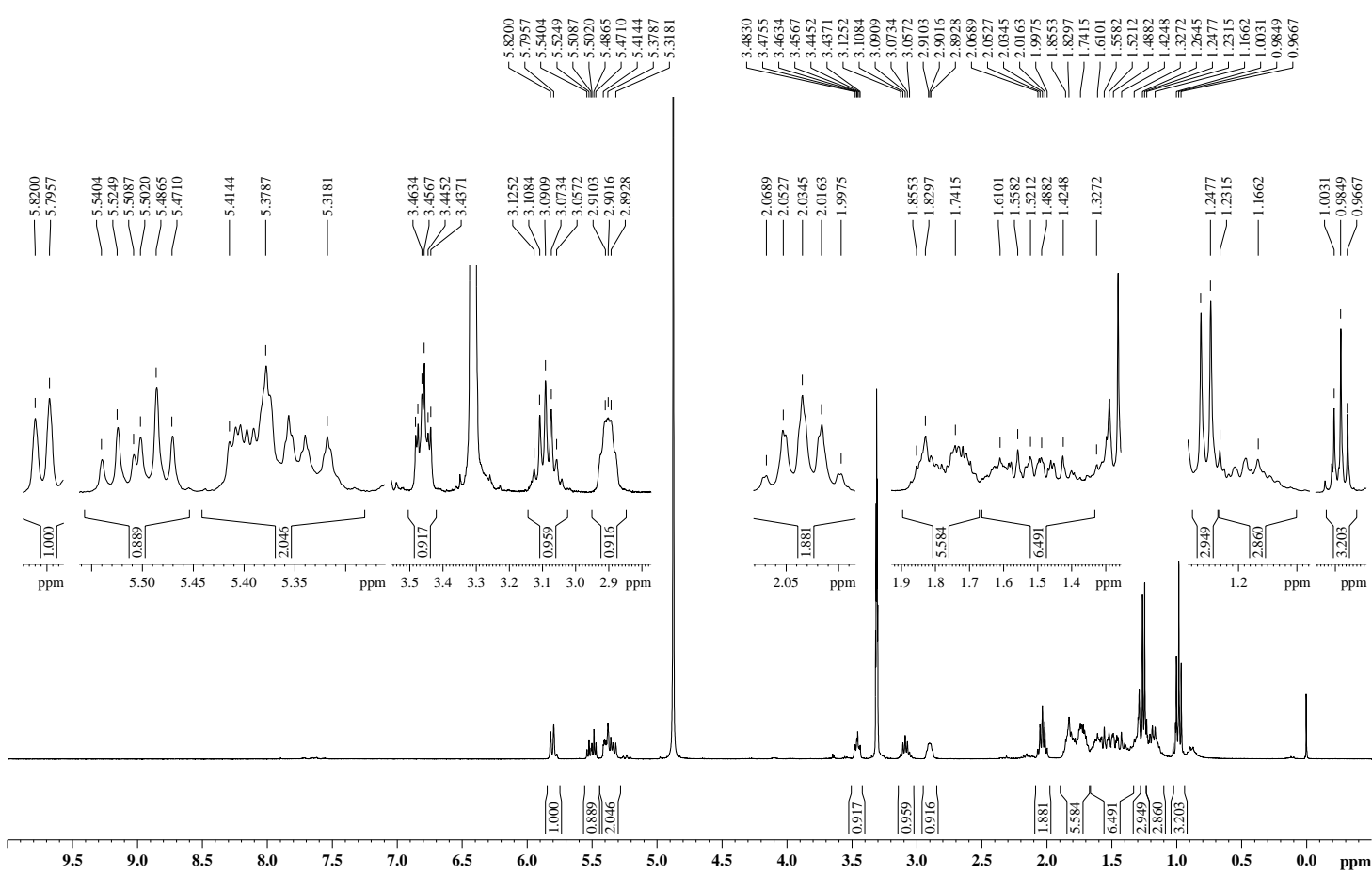

EKIV128 rescued +TFA

07.06.2007 MeOD
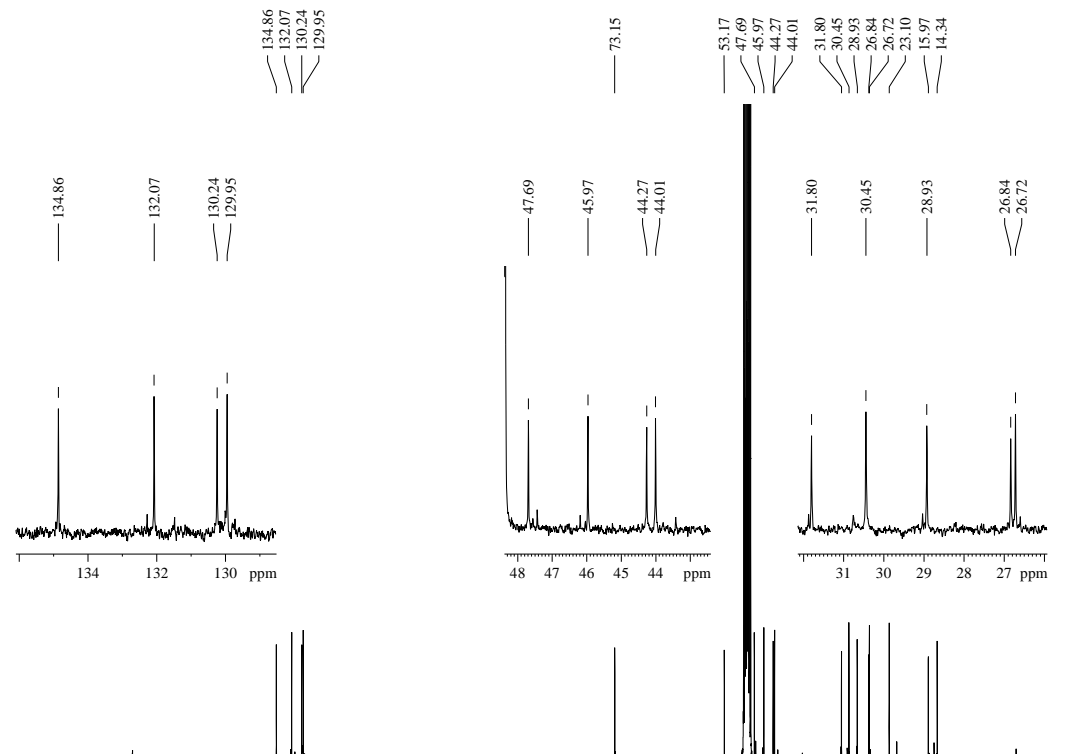

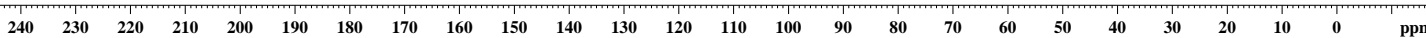




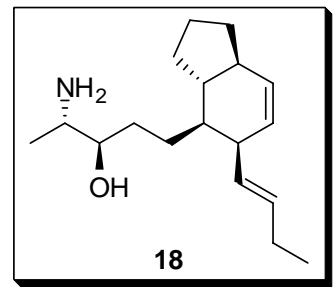

EKIV180 crude

04.09.2007 MeOD

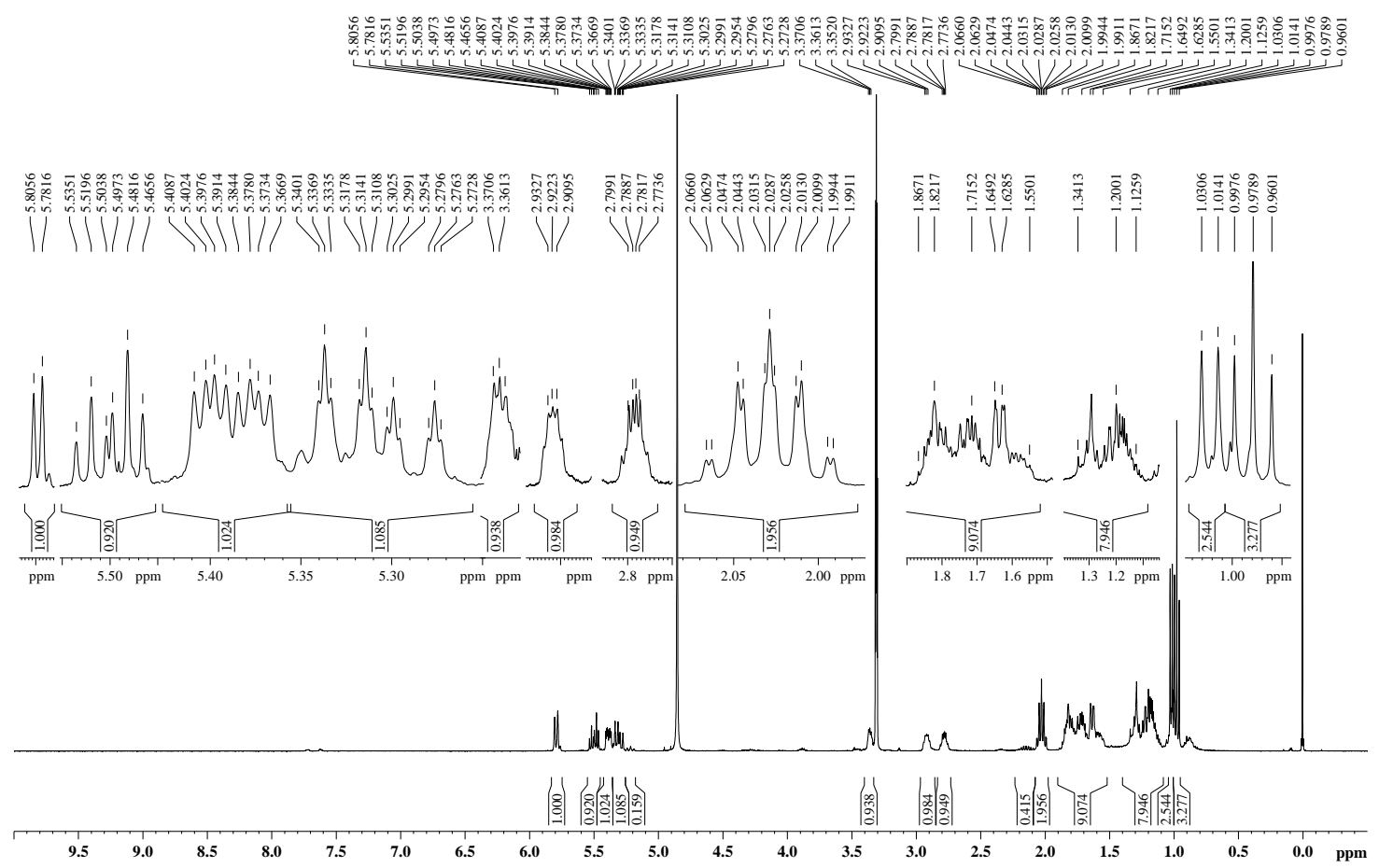

EKIV180 crude

04.09.2007 MeOD

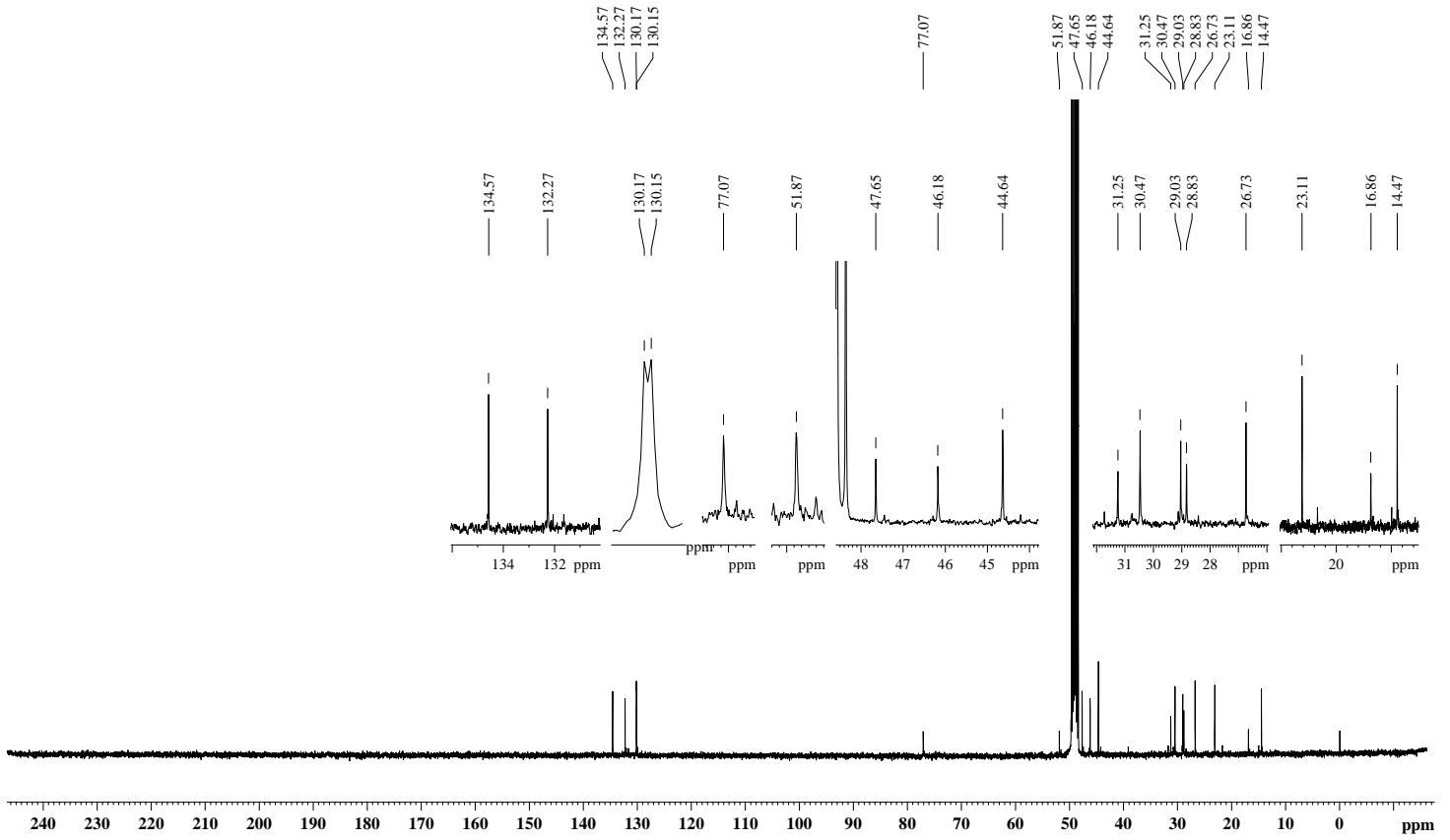




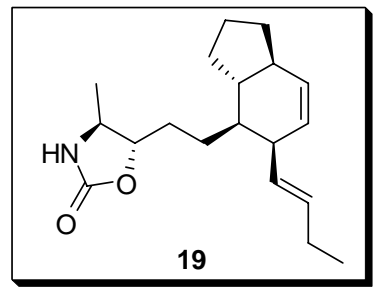

EKIV94 f8-11

03.04.2007 CDCl3

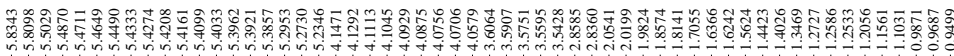

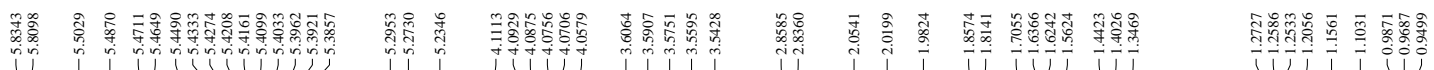

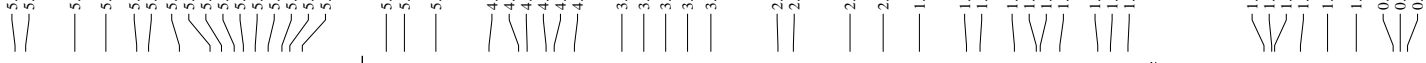
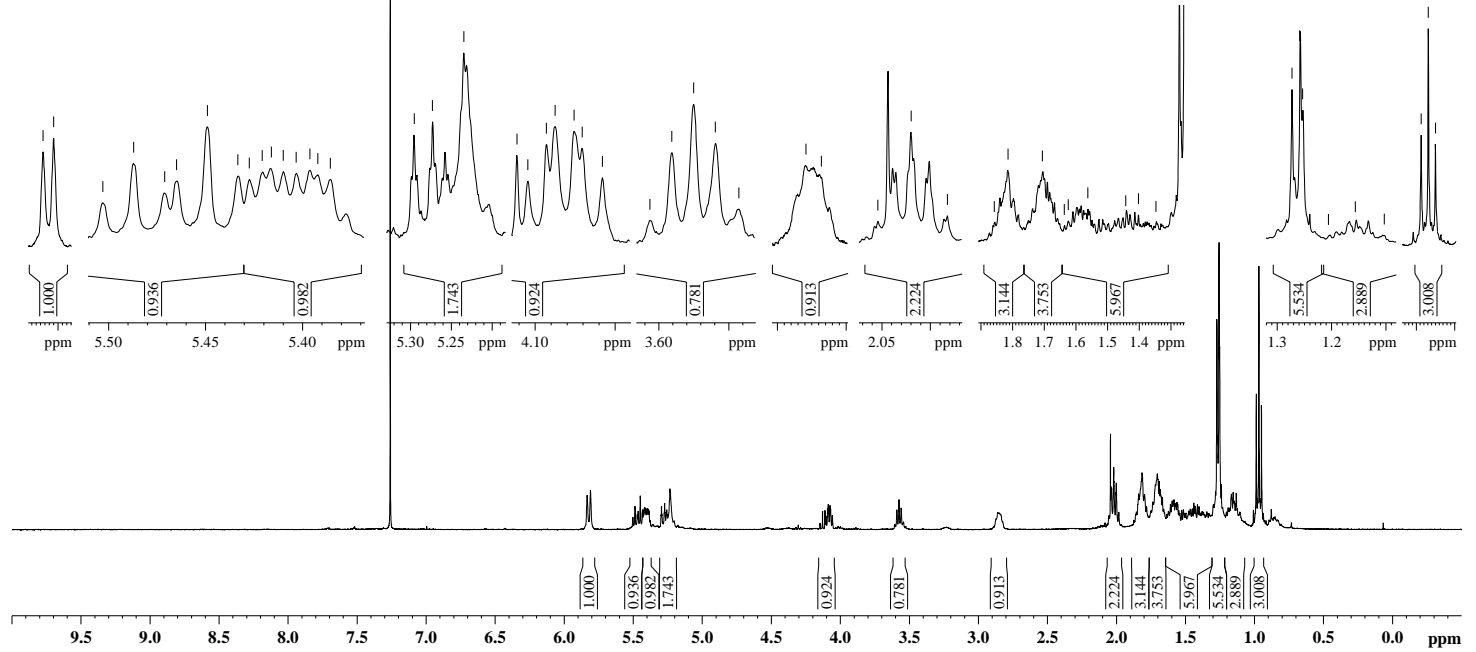

EKIV94 f8-11

11.04.2007 CDCl3

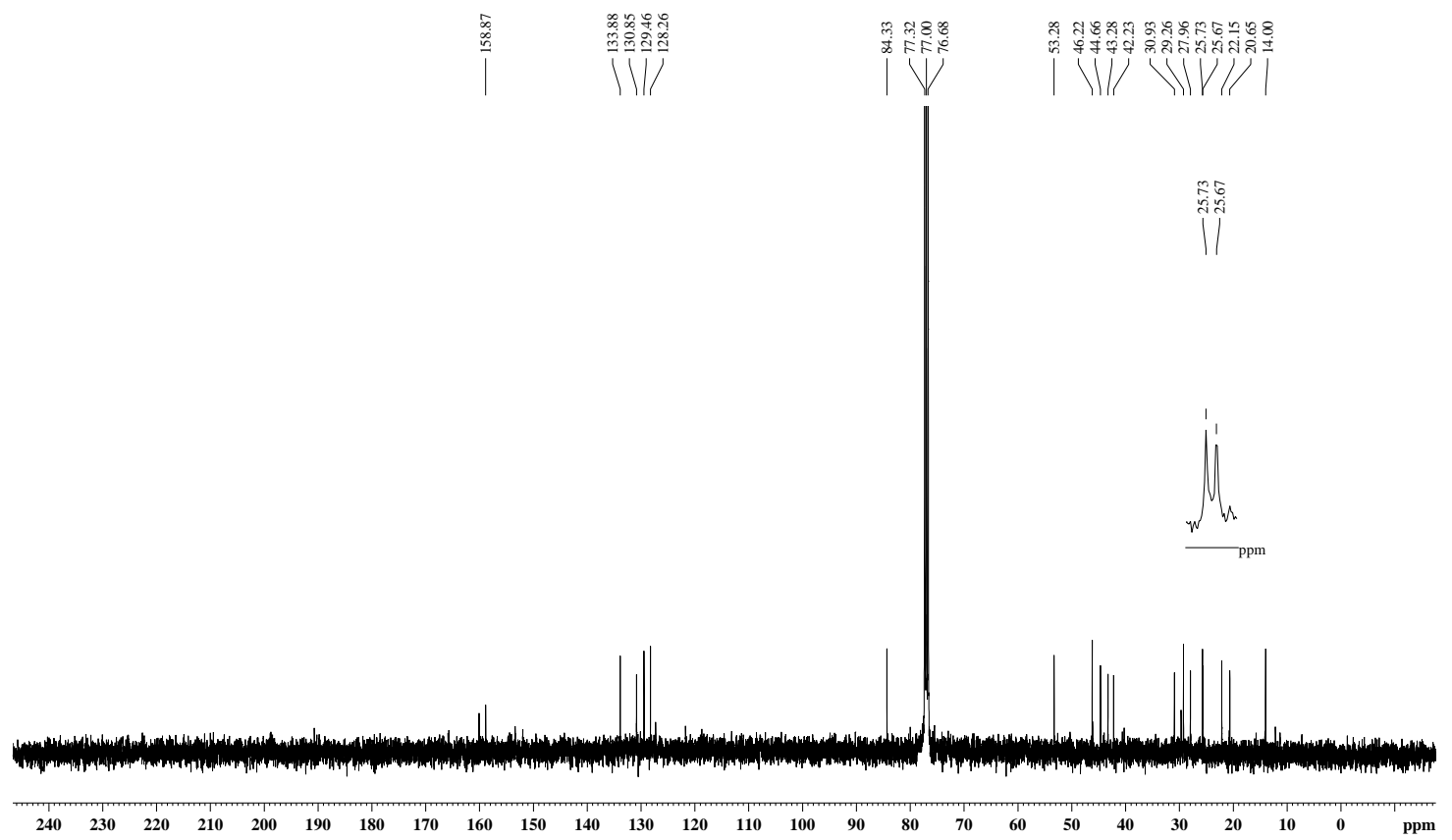




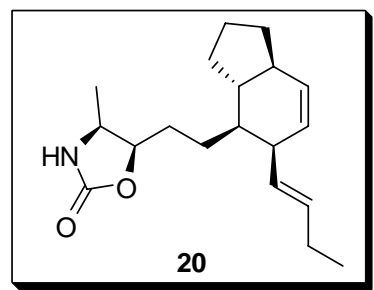

EKIV92 f5-15

29.03.2007 CDCl3

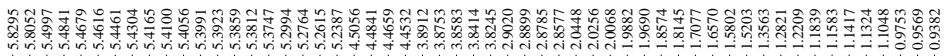

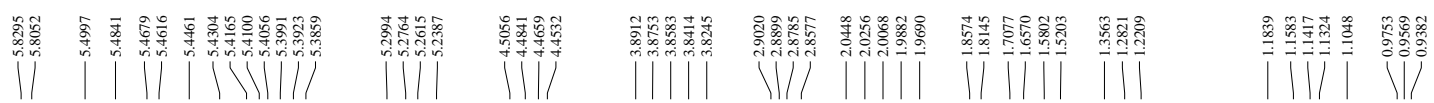
intor
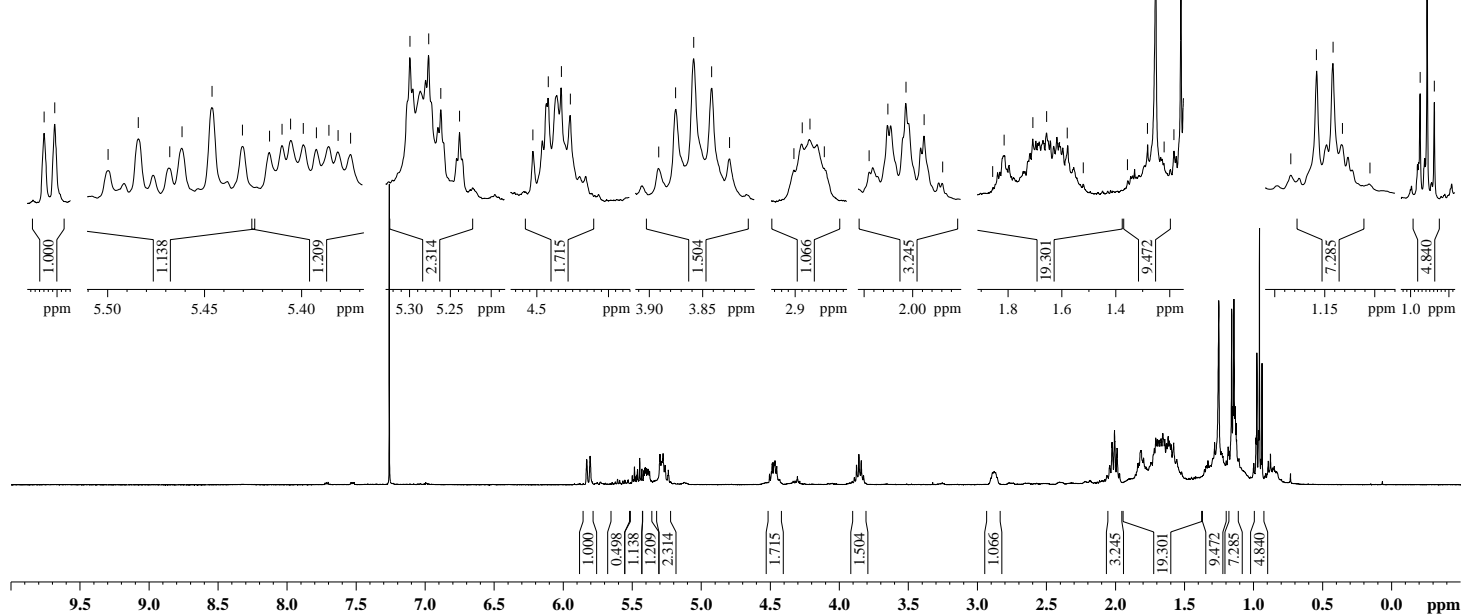

EKIV92 f5-15

29.03.2007 CDCl3

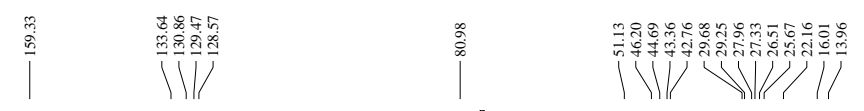

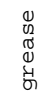

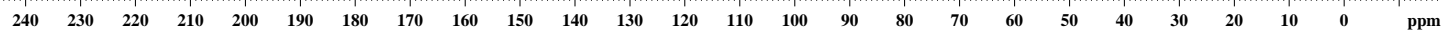

